\title{
Intermolecular Radical Carboaminohydroxylation of Olefins with Aryl Diazonium Salts and TEMPO
}

\author{
Markus R. Heinrich,* Alexander Wetzel and Marcel Kirschstein \\ Organische Chemie 1, Technische Universität München, Lichtenbergstr. 4, 85747 Garching, \\ Germany \\ Fax: +49-89-289-13329, Markus.Heinrich@ch.tum.de
}

\section{General experimental}

Solvents and reagents were degassed with argon prior to use in radical reactions. Infra-red spectra were recorded on a Fourier transform IR spectrometer. ${ }^{1} \mathrm{H}$ NMR spectra were recorded on 250, 360 and $500 \mathrm{MHz}$ spectrometers using $\mathrm{CDCl}_{3}, \mathrm{CD}_{3} \mathrm{OD}, \mathrm{CD}_{3} \mathrm{CN}$ and $\mathrm{C}_{6} \mathrm{D}_{6}$ as solvents referenced to TMS (0 ppm), $\mathrm{CHCl}_{3}$ (7.26 ppm), $\mathrm{CHD}_{2} \mathrm{OD}$ (3.31 ppm), $\mathrm{CHD}_{2} \mathrm{CN}$ (1.94 ppm) or $\mathrm{C}_{6} \mathrm{HD}_{5}(7.15 \mathrm{ppm})$. Chemical shifts are reported in parts per million (ppm). Coupling constants are in Hertz $(J \mathrm{~Hz})$. The following abbreviations are used for the description of signals: $s$ (singlet), $d$ (doublet), dd (double doublet), $t$ (triplet), q (quadruplet), $\mathrm{m}$ (multiplet). ${ }^{13} \mathrm{C}$ NMR spectra were recorded at 62.9 and $90.6 \mathrm{MHz}$ in $\mathrm{CDCl}_{3}, \mathrm{CD}_{3} \mathrm{OD}$, $\mathrm{CD}_{3} \mathrm{CN}$ and $\mathrm{C}_{6} \mathrm{D}_{6}$ using $\mathrm{CDCl}_{3}(77.0 \mathrm{ppm}), \mathrm{CD}_{3} \mathrm{OD}$ (49.5 ppm), $\mathrm{CD}_{3} \mathrm{CN}$ (1.24 ppm) and $\mathrm{C}_{6} \mathrm{D}_{6}$ $(128.0 \mathrm{ppm})$ as standard. Chemical shifts are given in parts per million (ppm). ${ }^{19} \mathrm{~F}$ NMR spectra were recorded at $235.3 \mathrm{MHz}$ using $\mathrm{CFCl}_{3}(0 \mathrm{ppm})$ as standard. Mass spectra were recorded using electron impact (EI). Analytical TLC was carried out on Merck silica gel plates using short wave $(254 \mathrm{~nm}) \mathrm{UV}$ light and $\mathrm{KMnO}_{4}$ to visualise components. Silica gel (Kieselgel 60, 40-63 $\mu \mathrm{m}$, Merck) was used for flash column chromatography. 


\section{Competition experiments}

In the competition experiments, the aryl diazonium salt (1mmol) was decomposed with $\mathrm{FeSO}_{4} \times 7 \mathrm{H}_{2} \mathrm{O}(3 \mathrm{mmol})$ in the presence of ethyl acrylate $(10 \mathrm{mmol})$, allyl acetate $(10 \mathrm{mmol})$ and TEMPO (3 mmol) in DMSO/water (5:1). After addition of ascorbic acid, aqueous workup and removal of excess olefin under reduced pressure, the product mixture was analyzed by 1H-NMR.

Similar results were obtained from the photochemical decomposition of bisphenyl carbodiazone $(\mathbf{8})(1 \mathrm{mmol})$ in the presence of ethyl acrylate $(10 \mathrm{mmol})$, allyl acetate (10 mmol) and TEMPO (3 mmol) in $\mathrm{CH}_{2} \mathrm{Cl}_{2}(5 \mathrm{~mL})$, although TEMPO-trapping of the aryl radical occured to an increased extent.

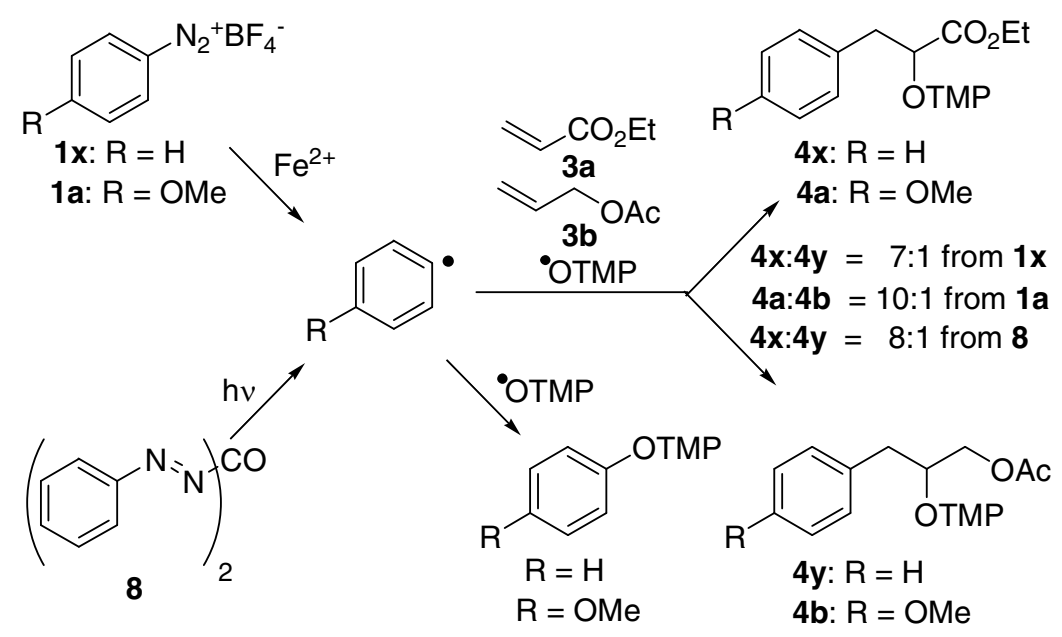

\section{3-Phenyl-2-(2,2,6,6-tetramethyl-piperidin-1-yloxy)-propionic acid ethyl ester (4x)}

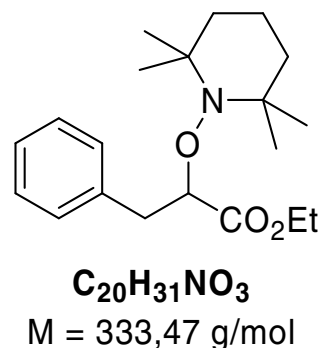

Light brown oil.

$R_{\mathrm{f}}: \quad 0.70\left(100 \% \mathrm{CH}_{2} \mathrm{Cl}_{2}\right)$

${ }^{1} \mathrm{H}-\mathrm{NMR}: \quad\left(360 \mathrm{MHz}, \mathrm{CDCl}_{3}\right): \delta=1.02\left(\mathrm{t},{ }^{3} J=7.2 \mathrm{~Hz}, 3 \mathrm{H}\right), 1.00-1.10(\mathrm{~m}, 2 \mathrm{H}), 1.13$ $(\mathrm{s}, 6 \mathrm{H}), 1.25(\mathrm{~s}, 6 \mathrm{H}), 1.35-1.58(\mathrm{~m}, 4 \mathrm{H}), 2.99\left(\mathrm{dd},{ }^{3} J=10.4 \mathrm{~Hz},{ }^{2} J=13.0\right.$ 
$\mathrm{Hz}, 1 \mathrm{H}), 3.27\left(\mathrm{dd},{ }^{3} J=5.4 \mathrm{~Hz},{ }^{2} J=13.0 \mathrm{~Hz}, 1 \mathrm{H}\right), 3.94\left(\mathrm{q},{ }^{3} J=7.2 \mathrm{~Hz}, 2 \mathrm{H}\right)$, $4.45\left(\mathrm{dd},{ }^{3} \mathrm{~J}=5.4 \mathrm{~Hz},{ }^{3} \mathrm{~J}=10.4 \mathrm{~Hz}, 1 \mathrm{H}\right), 7.13-7.26(\mathrm{~m}, 5 \mathrm{H})$.

${ }^{13} \mathrm{C}-\mathrm{NMR}: \quad\left(90.6 \mathrm{MHz}, \mathrm{CDCl}_{3}\right): \delta=13.8\left(\mathrm{CH}_{3}\right), 17.1\left(\mathrm{CH}_{2}\right), 20.1\left(\mathrm{CH}_{3}\right), 20.2\left(\mathrm{CH}_{3}\right)$, $33.0\left(\mathrm{CH}_{3}\right), 33.6\left(\mathrm{CH}_{3}\right), 39.8\left(\mathrm{CH}_{2}\right), 40.3\left(\mathrm{CH}_{2}\right), 59.3\left(\mathrm{C}_{\mathrm{q}}\right), 60.0\left(\mathrm{CH}_{2}\right), 60.7$ $\left(\mathrm{C}_{\mathrm{q}}\right), 86.7(\mathrm{CH}), 126.5(\mathrm{CH}), 128.2(2 \times \mathrm{CH}), 129.4(2 \times \mathrm{CH}), 136.1\left(\mathrm{C}_{\mathrm{q}}\right)$, $172.7\left(\mathrm{C}_{\mathrm{q}}\right)$.

ESI-MS: $\quad m / z=334\left[\mathrm{M}^{+}+\mathrm{H}\right]$.

HR-ESI-MS: $\quad \mathrm{C}_{20} \mathrm{H}_{32} \mathrm{NO}_{3}\left[\mathrm{M}^{+}+\mathrm{H}\right] \quad$ calcd.: 334.2377, found.: 334.2368 .

\section{Acetic acid 3-phenyl-2-(2,2,6,6-tetramethyl-piperidin-1-yloxy)-propyl ester (4y)}

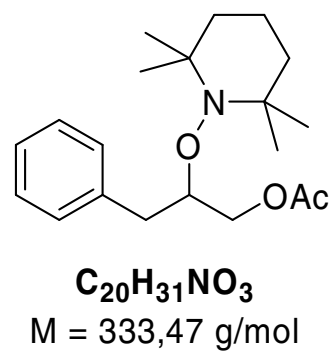

Colorless oil.

$R_{\mathrm{f}}: \quad 0.50\left(100 \% \mathrm{CH}_{2} \mathrm{Cl}_{2}\right)$

${ }^{1} \mathrm{H}-\mathrm{NMR}: \quad\left(360 \mathrm{MHz}, \mathrm{CDCl}_{3}\right): \delta=1.03(\mathrm{~s}, 3 \mathrm{H}), 1.10(\mathrm{~s}, 9 \mathrm{H}), 1.20-1.60(\mathrm{~m}, 6 \mathrm{H}), 2.06$ (s, 3H), $2.76\left(\mathrm{dd},{ }^{3} J=7.2 \mathrm{~Hz},{ }^{2} J=13.3 \mathrm{~Hz}, 1 \mathrm{H}\right), 3.13\left(\mathrm{dd},{ }^{3} J=5.0 \mathrm{~Hz},{ }^{2} J=\right.$ $13.3 \mathrm{~Hz}, 1 \mathrm{H}), 4.09-4.20$ (m, 3H), 7.18-7.29 (m, 5H).

${ }^{13} \mathrm{C}-\mathrm{NMR}: \quad\left(90.6 \mathrm{MHz}, \mathrm{CDCl}_{3}\right): \delta=14.4\left(\mathrm{CH}_{2}\right), 17.5\left(\mathrm{CH}_{3}\right), 17.7\left(\mathrm{CH}_{3}\right), 18.2\left(\mathrm{CH}_{3}\right)$, $30.9\left(\mathrm{CH}_{3}\right), 31.4\left(\mathrm{CH}_{3}\right), 34.5(\mathrm{CH}), 37.5\left(2 \times \mathrm{CH}_{2}\right), 57.0\left(\mathrm{C}_{\mathrm{q}}\right), 57.4\left(\mathrm{C}_{\mathrm{q}}\right), 61.3$ $\left(\mathrm{CH}_{2}\right), 77.9(\mathrm{CH}), 123.3(\mathrm{CH}), 125.4(2 \times \mathrm{CH}), 126.7(2 \times \mathrm{CH}), 135.7\left(\mathrm{C}_{\mathrm{q}}\right)$, $168.0\left(\mathrm{C}_{\mathrm{q}}\right)$.

APCI-MS: $\quad m / z=334\left[\mathrm{M}^{+}+\mathrm{H}\right]$.

HR-APCI -MS: $\mathrm{C}_{20} \mathrm{H}_{32} \mathrm{NO}_{3}\left[\mathrm{M}^{+}+\mathrm{H}\right] \quad$ calcd.: 334.2377, found.: 334.2374. 


\section{General procedure}

To a degassed 2:1 mixture of DMSO and water $(3 \mathrm{~mL})$ under argon were added the olefin (3 mmol for activated, $10 \mathrm{mmol}$ for nonactivated substrates), $\mathrm{FeSO}_{4} \times 7 \mathrm{H}_{2} \mathrm{O}(834 \mathrm{mg}, 3 \mathrm{mmol})$ and TEMPO (313 $\mathrm{mg}, 2 \mathrm{mmol})$. A solution of the arenediazonium salt $(1 \mathrm{mmol})$ in 2:1 DMSO-water $(1 \mathrm{~mL})$ was added dropwise over a period of 10 to $15 \mathrm{~min}$ and the resulting mixture was left to stir at r.t. for another $10 \mathrm{~min}$ before ascorbic acid (528 $\mathrm{mg}, 3 \mathrm{mmol})$ was added. After 10 more min, the mixture was diluted with water $(50 \mathrm{~mL})$ and extracted with $\mathrm{Et}_{2} \mathrm{O}(3 \times 30 \mathrm{~mL})$. The combined organic phases were washed with satd. $\mathrm{NaCl}$ and dried over $\mathrm{Na}_{2} \mathrm{SO}_{4}$. Concentration and purification by column chromatography furnished the carboaminohydroxylation products. Solvent mixtures for purification are given with the $R_{\mathrm{f}}$ values. Products were obtained as light brown oils or solids. 

ester (4a)

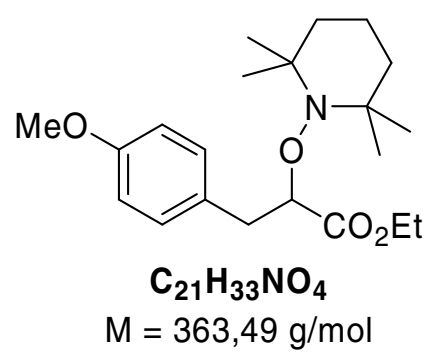

Light brown solid.

m.p.: $\quad 65-66{ }^{\circ} \mathrm{C}$

$R_{\mathrm{f}}: \quad 0.50\left(100 \% \mathrm{CH}_{2} \mathrm{Cl}_{2}\right)$

${ }^{1} \mathrm{H}-\mathrm{NMR}: \quad\left(360 \mathrm{MHz}, \mathrm{CDCl}_{3}\right): \delta=1.04(\mathrm{~s}, 3 \mathrm{H}), 1.05\left(\mathrm{t},{ }^{3} J=7.2 \mathrm{~Hz}, 3 \mathrm{H}\right), 1.13(\mathrm{~s}, 6 \mathrm{H})$, $1.20-1.55(\mathrm{~m}, 6 \mathrm{H}), 1.25(\mathrm{~s}, 3 \mathrm{H}), 2.93\left(\mathrm{dd},{ }^{3} J=10.4 \mathrm{~Hz},{ }^{2} J=13.3 \mathrm{~Hz}, 1 \mathrm{H}\right)$, $3.20\left(\mathrm{dd},{ }^{3} J=5.3 \mathrm{~Hz},{ }^{2} J=13.3 \mathrm{~Hz}, 1 \mathrm{H}\right), 3.76(\mathrm{~s}, 3 \mathrm{H}), 3.95(\mathrm{dq}, J=0.8 \mathrm{~Hz}$, $\left.{ }^{3} J=7.2 \mathrm{~Hz}, 2 \mathrm{H}\right), 4.39\left(\mathrm{dd},{ }^{3} J=5.3 \mathrm{~Hz},{ }^{3} J=10.4 \mathrm{~Hz}, 1 \mathrm{H}\right), 6.78\left(\mathrm{~d},{ }^{3} J=8.8\right.$ $\mathrm{Hz}, 2 \mathrm{H}), 7.07\left(\mathrm{~d},{ }^{3} \mathrm{~J}=8.8 \mathrm{~Hz}, 2 \mathrm{H}\right)$.

${ }^{13} \mathrm{C}-\mathrm{NMR}: \quad\left(90.6 \mathrm{MHz}, \mathrm{CDCl}_{3}\right): \delta=13.9\left(\mathrm{CH}_{3}\right), 17.1\left(\mathrm{CH}_{2}\right), 20.0\left(\mathrm{CH}_{3}\right), 20.2\left(\mathrm{CH}_{3}\right)$, $33.0\left(\mathrm{CH}_{3}\right), 33.5\left(\mathrm{CH}_{3}\right), 37.7\left(\mathrm{CH}_{2}\right), 40.3\left(2 \times \mathrm{CH}_{2}\right), 55.2\left(\mathrm{CH}_{3}\right), 59.4\left(\mathrm{C}_{\mathrm{q}}\right)$, $60.0\left(\mathrm{CH}_{2}\right), 60.4\left(\mathrm{C}_{\mathrm{q}}\right), 86.8(\mathrm{CH}), 113.7(2 \times \mathrm{CH}), 128.0\left(\mathrm{C}_{\mathrm{q}}\right), 130.4(2 \times \mathrm{CH})$, $158.3\left(\mathrm{C}_{\mathrm{q}}\right), 172.8\left(\mathrm{C}_{\mathrm{q}}\right)$.

ESI-MS: $\quad m / z=364\left[\mathrm{M}^{+}+\mathrm{H}\right]$.

HR-ESI-MS: $\quad \mathrm{C}_{21} \mathrm{H}_{34} \mathrm{NO}_{4}\left[\mathrm{M}^{+}+\mathrm{H}\right] \quad$ calcd.: 364.2482 , found.: 364.2472 .

Acetic acid 3-(4-methoxy-phenyl)-2-(2,2,6,6-tetramethyl-piperidin-1-yloxy)-propyl ester (4b)

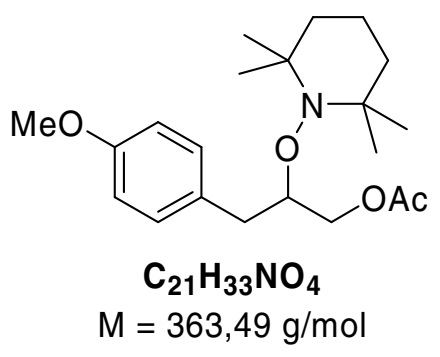

Light brown oil. 
$\left.R_{\mathrm{f}}: \quad 0.35100 \% \mathrm{CH}_{2} \mathrm{Cl}_{2}\right)$

${ }^{1} \mathrm{H}-\mathrm{NMR}: \quad\left(500 \mathrm{MHz}, \mathrm{CDCl}_{3}\right): \delta=1.03(\mathrm{~s}, 3 \mathrm{H}), 1.11(\mathrm{~s}, 6 \mathrm{H}), 1.14(\mathrm{~s}, 3 \mathrm{H}), 1.25-1.35$ (m, 1H), $1.35-1.60(\mathrm{~m}, 5 \mathrm{H}), 2.07(\mathrm{~s}, 3 \mathrm{H}), 2.71\left(\mathrm{dd},{ }^{3} J=7.8 \mathrm{~Hz},{ }^{2} J=13.8\right.$ $\mathrm{Hz}, 1 \mathrm{H}), 3.07\left(\mathrm{dd},{ }^{3} J=5.3 \mathrm{~Hz},{ }^{2} J=13.8 \mathrm{~Hz}, 1 \mathrm{H}\right), 3.77$ (s, 3H), 4.10-4.19 $(\mathrm{m}, 3 \mathrm{H}), 6.81\left(\mathrm{~d},{ }^{3} J=8.8 \mathrm{~Hz}, 2 \mathrm{H}\right), 7.11\left(\mathrm{~d},{ }^{3} J=8.8 \mathrm{~Hz}, 2 \mathrm{H}\right)$.

${ }^{13} \mathrm{C}-\mathrm{NMR}: \quad\left(90.6 \mathrm{MHz}, \mathrm{CDCl}_{3}\right): \delta=17.0\left(\mathrm{CH}_{2}\right), 20.1\left(\mathrm{CH}_{3}\right), 20.3\left(\mathrm{CH}_{3}\right), 20.7\left(\mathrm{CH}_{3}\right)$, $33.5\left(\mathrm{CH}_{3}\right), 34.0\left(\mathrm{CH}_{3}\right), 36.1\left(\mathrm{CH}_{2}\right), 40.1\left(2 \times \mathrm{CH}_{2}\right), 55.0\left(\mathrm{CH}_{3}\right), 59.6\left(\mathrm{C}_{\mathrm{q}}\right)$, $60.1\left(\mathrm{C}_{\mathrm{q}}\right), 63.9\left(\mathrm{CH}_{2}\right), 80.7(\mathrm{CH}), 113.5(2 \times \mathrm{CH}), 130.2\left(\mathrm{C}_{\mathrm{q}}\right), 130.2(2 \times \mathrm{CH})$, $157.8\left(\mathrm{C}_{\mathrm{q}}\right), 170.7\left(\mathrm{C}_{\mathrm{q}}\right)$.

APCI-MS: $\quad m / z=364\left[\mathrm{M}^{+}+\mathrm{H}\right]$.

HR-APCI -MS: $\mathrm{C}_{21} \mathrm{H}_{34} \mathrm{NO}_{4}\left[\mathrm{M}^{+}+\mathrm{H}\right] \quad$ calcd.: 364.2482, found.: 334.2477.

Acetic acid 2-(4-methoxy-phenyl)-1-(2,2,6,6-tetramethyl-piperidin-1-yloxy)-ethyl ester (4c)

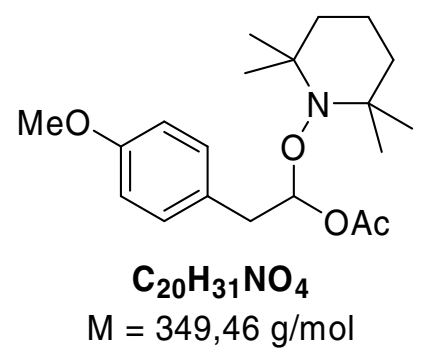

Light yellow oil.

$R_{\mathrm{f}}: \quad 0.50\left(100 \% \mathrm{CH}_{2} \mathrm{Cl}_{2}\right)$

${ }^{1} \mathrm{H}-\mathrm{NMR}: \quad\left(360 \mathrm{MHz}, \mathrm{CDCl}_{3}\right): \delta=1.00(\mathrm{~s}, 3 \mathrm{H}), 1.04(\mathrm{~s}, 3 \mathrm{H}), 1.08(\mathrm{~s}, 3 \mathrm{H}), 1.12(\mathrm{~s}, 3 \mathrm{H})$, $1.22-1.63(\mathrm{~m}, 6 \mathrm{H}), 1.96(\mathrm{~s}, 3 \mathrm{H}), 2.90\left(\mathrm{dd},{ }^{3} \mathrm{~J}=5.4 \mathrm{~Hz},{ }^{2} J=14.0 \mathrm{~Hz}, 1 \mathrm{H}\right)$, $2.99\left(\mathrm{dd},{ }^{3} J=5.4 \mathrm{~Hz},{ }^{2} J=14.0 \mathrm{~Hz}, 1 \mathrm{H}\right), 3.78(\mathrm{~s}, 3 \mathrm{H}), 6.29\left(\right.$, t“" ${ }^{3} J=5.4 \mathrm{~Hz}$, $1 \mathrm{H}), 6.81\left(\mathrm{~d},{ }^{3} J=7.2 \mathrm{~Hz}, 2 \mathrm{H}\right), 7.16\left(\mathrm{~d},{ }^{3} J=7.2 \mathrm{~Hz}, 2 \mathrm{H}\right)$.

${ }^{13} \mathrm{C}-\mathrm{NMR}: \quad\left(90.6 \mathrm{MHz}, \mathrm{CDCl}_{3}\right): \delta=17.6\left(\mathrm{CH}_{2}\right), 20.1\left(\mathrm{CH}_{3}\right), 20.4\left(\mathrm{CH}_{3}\right), 21.3\left(\mathrm{CH}_{3}\right)$, $32.9\left(\mathrm{CH}_{3}\right), 33.7\left(\mathrm{CH}_{3}\right), 39.1\left(\mathrm{CH}_{2}\right), 40.0\left(\mathrm{CH}_{2}\right), 40.2\left(\mathrm{CH}_{2}\right), 55.2\left(\mathrm{CH}_{3}\right)$, $59.1\left(\mathrm{C}_{\mathrm{q}}\right), 60.8\left(\mathrm{C}_{\mathrm{q}}\right), 101.2(\mathrm{CH}), 113.5(2 \times \mathrm{CH}), 127.7\left(\mathrm{C}_{\mathrm{q}}\right), 131.0(2 \times \mathrm{CH})$, $158.3\left(\mathrm{C}_{\mathrm{q}}\right), 169.9\left(\mathrm{C}_{\mathrm{q}}\right)$.

APCI-MS: $\quad m / z=350\left[\mathrm{M}^{+}+\mathrm{H}\right]$.

HR-APCI -MS: $\mathrm{C}_{20} \mathrm{H}_{32} \mathrm{NO}_{4}\left[\mathrm{M}^{+}+\mathrm{H}\right] \quad$ calcd.: 350.2325 , found.: 350.2319 . 
(4d)

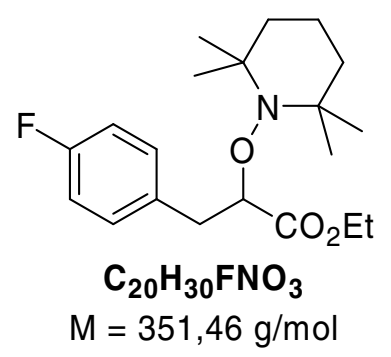

Light brown solid.

m.p.: $\quad 60-61{ }^{\circ} \mathrm{C}$

$R_{\mathrm{f}}: \quad 0.70\left(100 \% \mathrm{CH}_{2} \mathrm{Cl}_{2}\right)$

${ }^{1} \mathrm{H}-\mathrm{NMR}: \quad\left(250 \mathrm{MHz}, \mathrm{CDCl}_{3}\right): \delta=1.03(\mathrm{~s}, 3 \mathrm{H}), 1.04\left(\mathrm{t},{ }^{3} J=7.3 \mathrm{~Hz}, 3 \mathrm{H}\right), 1.12(\mathrm{~s}, 6 \mathrm{H})$, $1.20-1.55(\mathrm{~m}, 6 \mathrm{H}), 1.23(\mathrm{~s}, 3 \mathrm{H}), 2.96\left(\mathrm{dd},{ }^{3} J=10.3 \mathrm{~Hz},{ }^{2} J=13.3 \mathrm{~Hz}, 1 \mathrm{H}\right)$, $3.22\left(\mathrm{dd},{ }^{3} J=5.5 \mathrm{~Hz},{ }^{2} J=13.3 \mathrm{~Hz}, 1 \mathrm{H}\right), 3.95\left(\mathrm{q},{ }^{3} J=7.3 \mathrm{~Hz}, 2 \mathrm{H}\right), 4.40$ (dd, $\left.{ }^{3} J=5.5 \mathrm{~Hz},{ }^{3} J=10.3 \mathrm{~Hz}, 1 \mathrm{H}\right), 6.93\left(\mathrm{dd},{ }^{3} J_{\mathrm{HF}}=8.8 \mathrm{~Hz},{ }^{3} J=8.8 \mathrm{~Hz}, 2 \mathrm{H}\right)$, $7.11\left(\mathrm{dd},{ }^{4} J_{\mathrm{HF}}=5.5 \mathrm{~Hz},{ }^{3} \mathrm{~J}=8.8 \mathrm{~Hz}, 2 \mathrm{H}\right)$.

${ }^{13} \mathrm{C}-\mathrm{NMR}: \quad\left(62.9 \mathrm{MHz}, \mathrm{CDCl}_{3}\right): \delta=13.9\left(\mathrm{CH}_{3}\right), 17.0\left(\mathrm{CH}_{2}\right), 20.0\left(\mathrm{CH}_{3}\right), 20.2\left(\mathrm{CH}_{3}\right)$, $33.0\left(\mathrm{CH}_{3}\right), 33.6\left(\mathrm{CH}_{3}\right), 37.7\left(\mathrm{CH}_{2}\right), 40.2\left(2 \times \mathrm{CH}_{2}\right), 59.4\left(\mathrm{C}_{\mathrm{q}}\right), 60.1\left(\mathrm{CH}_{2}\right)$, $60.4\left(\mathrm{C}_{\mathrm{q}}\right), 86.5\left(\mathrm{~d},{ }^{5} J_{\mathrm{CF}}=1.3 \mathrm{~Hz}, \mathrm{CH}\right), 115.0\left(\mathrm{~d},{ }^{2} J_{\mathrm{CF}}=21.3 \mathrm{~Hz}, 2 \times \mathrm{CH}\right)$, $130.9\left(\mathrm{~d},{ }^{3} J_{\mathrm{CF}}=7.9 \mathrm{~Hz}, 2 \times \mathrm{CH}\right), 131.8\left(\mathrm{~d},{ }^{4} J_{\mathrm{CF}}=3.3 \mathrm{~Hz}, \mathrm{C}_{\mathrm{q}}\right), 161.7\left(\mathrm{~d},{ }^{1} J_{\mathrm{CF}}=\right.$ $\left.244.6 \mathrm{~Hz}, \mathrm{C}_{\mathrm{q}}\right), 172.6\left(\mathrm{C}_{\mathrm{q}}\right)$.

${ }^{19}$ F-NMR: $\quad\left(235.3 \mathrm{MHz}, \mathrm{CDCl}_{3}\right): \delta=-116.9$.

ESI-MS: $\quad m / z=352\left[\mathrm{M}^{+}+\mathrm{H}\right]$.

HR-ESI-MS: $\quad \mathrm{C}_{20} \mathrm{H}_{31} \mathrm{FNO}_{3}\left[\mathrm{M}^{+}+\mathrm{H}\right]$ calcd.: 352.2282, found.: 352.2273 .

Acetic acid 3-(4-fluoro-phenyl)-2-(2,2,6,6-tetramethyl-piperidin-1-yloxy)-propyl ester (4e)

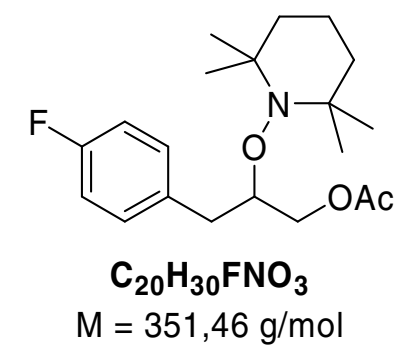


Light brown oil.

$R_{\mathrm{f}}:$

$0.50\left(100 \% \mathrm{CH}_{2} \mathrm{Cl}_{2}\right)$

${ }^{1} \mathrm{H}-\mathrm{NMR}: \quad\left(250 \mathrm{MHz}, \mathrm{CDCl}_{3}\right): \delta=1.01(\mathrm{~s}, 3 \mathrm{H}), 1.07(\mathrm{~s}, 3 \mathrm{H}), 1.09(\mathrm{~s}, 6 \mathrm{H}), 1.20-1.60$ (m, 6H), 2.07 (s, 3H), $2.75\left(\mathrm{dd},{ }^{3} J=6.8 \mathrm{~Hz},{ }^{2} J=13.8 \mathrm{~Hz}, 1 \mathrm{H}\right), 3.05\left(\mathrm{dd},{ }^{3} J\right.$ $\left.=5.5 \mathrm{~Hz},{ }^{2} J=13.8 \mathrm{~Hz}, 1 \mathrm{H}\right), 4.06-4.25(\mathrm{~m}, 3 \mathrm{H}), 6.96\left(\mathrm{dd},{ }^{3} J_{\mathrm{HF}}=8.8 \mathrm{~Hz},{ }^{3} \mathrm{~J}=\right.$ $8.8 \mathrm{~Hz}, 2 \mathrm{H}), 7.16\left(\mathrm{dd},{ }^{4} J_{\mathrm{HF}}=5.5 \mathrm{~Hz},{ }^{3} \mathrm{~J}=8.8 \mathrm{~Hz}, 2 \mathrm{H}\right)$.

${ }^{13} \mathrm{C}-\mathrm{NMR}: \quad\left(62.9 \mathrm{MHz}, \mathrm{CDCl}_{3}\right): \delta=17.2\left(\mathrm{CH}_{2}\right), 20.3\left(\mathrm{CH}_{3}\right), 20.5\left(\mathrm{CH}_{3}\right), 20.9\left(\mathrm{CH}_{3}\right)$, $33.7\left(\mathrm{CH}_{3}\right), 34.2\left(\mathrm{CH}_{3}\right), 36.6\left(\mathrm{CH}_{2}\right), 40.3\left(2 \times \mathrm{CH}_{2}\right), 59.8\left(\mathrm{C}_{\mathrm{q}}\right), 60.4\left(\mathrm{C}_{\mathrm{q}}\right), 64.0$ $\left(\mathrm{CH}_{2}\right), 80.5\left(\mathrm{~d},{ }^{5} J_{\mathrm{CF}}=1.3 \mathrm{~Hz}, \mathrm{CH}\right), 115.0\left(\mathrm{~d},{ }^{2} J_{\mathrm{CF}}=21.1 \mathrm{~Hz}, 2 \times \mathrm{CH}\right), 130.9$ $\left(\mathrm{d},{ }^{3} J_{\mathrm{CF}}=7.8 \mathrm{~Hz}, 2 \times \mathrm{CH}\right), 134.1\left(\mathrm{~d},{ }^{4} J_{\mathrm{CF}}=3.2 \mathrm{~Hz}, \mathrm{C}_{\mathrm{q}}\right), 161.5\left(\mathrm{~d},{ }^{1} J_{\mathrm{CF}}=243.9\right.$ $\left.\mathrm{Hz}, \mathrm{C}_{\mathrm{q}}\right), 170.8\left(\mathrm{C}_{\mathrm{q}}\right)$.

${ }^{19}$ F-NMR: $\quad\left(235.3 \mathrm{MHz}, \mathrm{CDCl}_{3}\right): \delta=-117.7$.

APCI-MS: $\quad m / z=352\left[\mathrm{M}^{+}+\mathrm{H}\right]$.

HR-APCI-MS: $\mathrm{C}_{20} \mathrm{H}_{31} \mathrm{FNO}_{3}\left[\mathrm{M}^{+}+\mathrm{H}\right]$ calcd.: 352.2282, found.: 352.2276.

\section{Acetic acid 2-(4-fluoro-phenyl)-1-(2,2,6,6-tetramethyl-piperidin-1-yloxy)-ethyl ester (4f)}

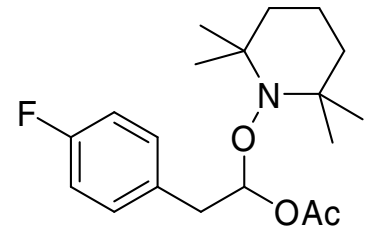

$\mathrm{C}_{19} \mathrm{H}_{28} \mathrm{FNO}_{3}$

$\mathrm{M}=337,43 \mathrm{~g} / \mathrm{mol}$

Light brown oil.

$R_{\mathrm{f}}: \quad 0.60\left(100 \% \mathrm{CH}_{2} \mathrm{Cl}_{2}\right)$

${ }^{1} \mathrm{H}-\mathrm{NMR}: \quad\left(250 \mathrm{MHz}, \mathrm{CDCl}_{3}\right): \delta=0.99(\mathrm{~s}, 3 \mathrm{H}), 1.02(\mathrm{~s}, 3 \mathrm{H}), 1.07(\mathrm{~s}, 3 \mathrm{H}), 1.11(\mathrm{~s}, 3 \mathrm{H})$, 1.20-1.60 (m, 6H), $1.96(\mathrm{~s}, 3 \mathrm{H}), 2.93\left(\mathrm{dd},{ }^{3} \mathrm{~J}=5.5 \mathrm{~Hz},{ }^{2} J=14.1 \mathrm{~Hz}, 1 \mathrm{H}\right)$, $3.01\left(\mathrm{dd},{ }^{3} J=5.5 \mathrm{~Hz},{ }^{2} J=14.1 \mathrm{~Hz}, 1 \mathrm{H}\right), 6.29\left(, \mathrm{t}^{\star},{ }^{3} J=5.5 \mathrm{~Hz}, 1 \mathrm{H}\right), 6.96$ $\left(\mathrm{dd},{ }^{3} J_{\mathrm{HF}}=8.8 \mathrm{~Hz},{ }^{3} \mathrm{~J}=8.8 \mathrm{~Hz}, 2 \mathrm{H}\right), 7.21\left(\mathrm{dd},{ }^{4} J_{\mathrm{HF}}=5.5 \mathrm{~Hz},{ }^{3} \mathrm{~J}=8.8 \mathrm{~Hz}\right.$, $2 \mathrm{H})$.

${ }^{13} \mathrm{C}-\mathrm{NMR}: \quad\left(62.9 \mathrm{MHz}, \mathrm{CDCl}_{3}\right): \delta=17.0\left(\mathrm{CH}_{2}\right), 20.1\left(\mathrm{CH}_{3}\right), 20.4\left(\mathrm{CH}_{3}\right), 21.3\left(\mathrm{CH}_{3}\right)$, $32.9\left(\mathrm{CH}_{3}\right), 33.8\left(\mathrm{CH}_{3}\right), 39.2\left(\mathrm{CH}_{2}\right), 40.0\left(\mathrm{CH}_{2}\right), 40.2\left(\mathrm{CH}_{2}\right), 59.2\left(\mathrm{C}_{\mathrm{q}}\right), 60.8$ $\left(\mathrm{C}_{\mathrm{q}}\right), 100.8\left(\mathrm{~d},{ }^{5} J_{\mathrm{CF}}=1.3 \mathrm{~Hz}, \mathrm{CH}\right), 114.9\left(\mathrm{~d},{ }^{2} J_{\mathrm{CF}}=21.2 \mathrm{~Hz}, 2 \times \mathrm{CH}\right), 131.4$ 
$\left(\mathrm{d},{ }^{4} J_{\mathrm{CF}}=3.3 \mathrm{~Hz}, \mathrm{C}_{\mathrm{q}}\right), 131.5\left(\mathrm{~d},{ }^{3} J_{\mathrm{CF}}=7.9 \mathrm{~Hz}, 2 \times \mathrm{CH}\right), 161.8\left(\mathrm{~d},{ }^{1} J_{\mathrm{CF}}=244.4\right.$ $\left.\mathrm{Hz}, \mathrm{C}_{\mathrm{q}}\right), 169.9\left(\mathrm{C}_{\mathrm{q}}\right)$.

${ }^{19}$ F-NMR: $\quad\left(235.3 \mathrm{MHz}, \mathrm{CDCl}_{3}\right): \delta=-117.0$.

EI-MS: $\quad m / z(\%)=337(0.5)\left[\mathrm{M}^{+}\right], 199(22), 184(25), 157(84), 142(100), 109(18)$, 83 (17), 69 (15), 55 (21).

HR-ESI-MS: $\quad \mathrm{C}_{19} \mathrm{H}_{29} \mathrm{FNO}_{3}\left[\mathrm{M}^{+}+\mathrm{H}\right] \quad$ calcd.: 338.2126 , found.: 338.2118 .

\section{2-[2-Ethoxycarbonyl-2-(2,2,6,6-tetramethyl-piperidin-1-yloxy)-ethyl]-benzoic acid} methyl ester (4g)

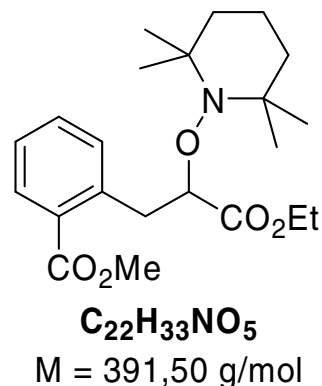

Light yellow oil.

$R_{\mathrm{f}}: \quad 0.50\left(1: 100\right.$ acetone- $\left.-\mathrm{CH}_{2} \mathrm{Cl}_{2}\right)$

${ }^{1} \mathrm{H}-\mathrm{NMR}: \quad\left(250 \mathrm{MHz}, \mathrm{CDCl}_{3}\right): \delta=1.00\left(\mathrm{t},{ }^{3} J=7.1 \mathrm{~Hz}, 3 \mathrm{H}\right), 1.03(\mathrm{~s}, 3 \mathrm{H}), 1.04(\mathrm{~s}, 3 \mathrm{H})$, $1.11(\mathrm{~s}, 6 \mathrm{H}), 1.20-1.55(\mathrm{~m}, 6 \mathrm{H}), 3.18\left(\mathrm{dd},{ }^{3} \mathrm{~J}=8.6 \mathrm{~Hz},{ }^{2} J=12.9 \mathrm{~Hz}, 1 \mathrm{H}\right)$, $3.70-3.95$ (m, 3H), 3.88 (s, 3H), 4.59 (dd, $\left.{ }^{3} J=7.2 \mathrm{~Hz},{ }^{3} J=8.6 \mathrm{~Hz}, 1 \mathrm{H}\right)$, 7.22-7.29 (m, 2H), 7.35-7.42 (m, 1H), 7.84-7.87 (m, 1H).

${ }^{13} \mathrm{C}-\mathrm{NMR}: \quad\left(90.6 \mathrm{MHz}, \mathrm{CDCl}_{3}\right): \delta=13.8\left(\mathrm{CH}_{3}\right), 17.1\left(\mathrm{CH}_{2}\right), 20.1\left(\mathrm{CH}_{3}\right), 20.2\left(\mathrm{CH}_{3}\right)$, $33.0\left(\mathrm{CH}_{3}\right), 33.5\left(\mathrm{CH}_{3}\right), 37.1\left(\mathrm{CH}_{2}\right), 40.3\left(2 \times \mathrm{CH}_{2}\right), 52.0\left(\mathrm{CH}_{3}\right), 59.4\left(\mathrm{C}_{\mathrm{q}}\right)$, $59.9\left(\mathrm{CH}_{2}\right), 60.3\left(\mathrm{C}_{\mathrm{q}}\right), 85.4(\mathrm{CH}), 126.7(\mathrm{CH}), 130.4\left(\mathrm{C}_{\mathrm{q}}\right), 130.6(\mathrm{CH}), 131.6$ $(\mathrm{CH}), 132.7(\mathrm{CH}), 137.8\left(\mathrm{C}_{\mathrm{q}}\right), 167.7\left(\mathrm{C}_{\mathrm{q}}\right), 172.7\left(\mathrm{C}_{\mathrm{q}}\right)$.

ESI-MS: $\quad m / z=392\left[\mathrm{M}^{+}+\mathrm{H}\right]$.

HR-ESI-MS: $\quad \mathrm{C}_{22} \mathrm{H}_{34} \mathrm{NO}_{5}\left[\mathrm{M}^{+}+\mathrm{H}\right] \quad$ calcd.: 392.2431, found.: 392.2418. 
(4h)

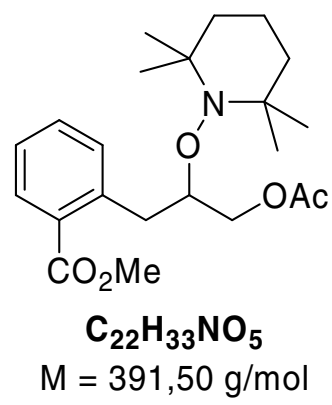

Light brown oil.

$R_{\mathrm{f}}: \quad 0.35\left(1: 100\right.$ acetone $\left.-\mathrm{CH}_{2} \mathrm{Cl}_{2}\right)$

${ }^{1} \mathrm{H}-\mathrm{NMR}: \quad\left(500 \mathrm{MHz}, \mathrm{CDCl}_{3}\right): \delta=0.80(\mathrm{~s}, 3 \mathrm{H}), 0.85(\mathrm{~s}, 3 \mathrm{H}), 1.05$ (s, 3H), 1.09 (s, 3H), $1.20-1.27(\mathrm{~m}, 2 \mathrm{H}), 1.36-1.52(\mathrm{~m}, 4 \mathrm{H}), 2.03(\mathrm{~s}, 3 \mathrm{H}), 3.21\left(\mathrm{dd},{ }^{3} \mathrm{~J}=8.0 \mathrm{~Hz}\right.$, $\left.{ }^{2} J=13.3 \mathrm{~Hz}, 1 \mathrm{H}\right), 3.30\left(\mathrm{dd},{ }^{3} J=5.2 \mathrm{~Hz},{ }^{2} J=13.3 \mathrm{~Hz}, 1 \mathrm{H}\right), 3.84(\mathrm{~s}, 3 \mathrm{H})$, $4.16\left(\mathrm{dd},{ }^{3} J=5.0 \mathrm{~Hz},{ }^{2} J=11.0 \mathrm{~Hz}, 1 \mathrm{H}\right), 4.22-4.27(\mathrm{~m}, 1 \mathrm{H}), 4.30\left(\mathrm{dd},{ }^{3} J=\right.$ $\left.3.5 \mathrm{~Hz},{ }^{3} J=11.0 \mathrm{~Hz}, 1 \mathrm{H}\right), 7.22\left(, \mathrm{t}\right.$ “", $\left.{ }^{3} J=7.5 \mathrm{~Hz}, 1 \mathrm{H}\right), 7.32\left(\mathrm{~d},{ }^{3} J=7.5 \mathrm{~Hz}\right.$, $1 \mathrm{H}), 7.38\left(\right.$, t“", $\left.{ }^{3} J=7.5 \mathrm{~Hz}, 1 \mathrm{H}\right), 7.83\left(\mathrm{~d},{ }^{3} J=7.5 \mathrm{~Hz}, 1 \mathrm{H}\right)$.

${ }^{13} \mathrm{C}-\mathrm{NMR}: \quad\left(62.9 \mathrm{MHz}, \mathrm{CDCl}_{3}\right): \delta=17.0\left(\mathrm{CH}_{2}\right), 20.0\left(\mathrm{CH}_{3}\right), 20.3\left(\mathrm{CH}_{3}\right), 20.8\left(\mathrm{CH}_{3}\right)$, $33.2\left(\mathrm{CH}_{3}\right), 33.9\left(\mathrm{CH}_{3}\right), 35.7\left(\mathrm{CH}_{2}\right), 40.1\left(\mathrm{CH}_{2}\right), 40.3\left(\mathrm{CH}_{2}\right), 51.6\left(\mathrm{CH}_{3}\right)$, $59.3\left(\mathrm{C}_{\mathrm{q}}\right), 60.2\left(\mathrm{C}_{\mathrm{q}}\right), 64.7\left(\mathrm{CH}_{2}\right), 79.8(\mathrm{CH}), 126.0(\mathrm{CH}), 130.0\left(\mathrm{C}_{\mathrm{q}}\right), 130.3$ $(\mathrm{CH}), 131.2(\mathrm{CH}), 132.5(\mathrm{CH}), 140.3\left(\mathrm{C}_{\mathrm{q}}\right), 167.8\left(\mathrm{C}_{\mathrm{q}}\right), 170.7\left(\mathrm{C}_{\mathrm{q}}\right)$.

EI-MS: $\quad m / z(\%)=391(0.5)\left[\mathrm{M}^{+}+\mathrm{H}\right], 236(11), 235(74), 176(11), 175(100), 161$ (12), 157 (12), 156 (7), 143 (13), 142 (64), 131 (26), 114 (8), 91 (10), 83 (10).

ESI-MS: $\quad m / z=392\left[\mathrm{M}^{+}+\mathrm{H}\right]$.

HR-ESI-MS: $\quad \mathrm{C}_{22} \mathrm{H}_{34} \mathrm{NO}_{5}\left[\mathrm{M}^{+}+\mathrm{H}\right] \quad$ calcd.: 392.2431 , found.: 392.2418 .

\section{2-[2-Acetoxy-2-(2,2,6,6-tetramethyl-piperidin-1-yloxy)-ethyl]-benzoic acid methyl ester} (4i)

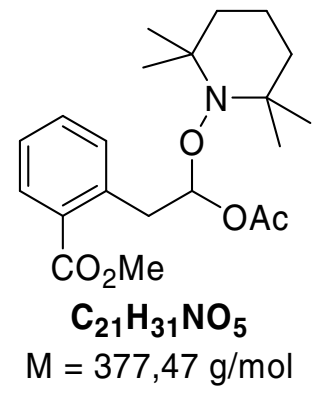


Light yellow oil.

$R_{\mathrm{f}}: \quad 0.5(1: 4$ EtOAc-pentane $)$

${ }^{1} \mathrm{H}-\mathrm{NMR}: \quad\left(250 \mathrm{MHz}, \mathrm{CDCl}_{3}\right): \delta=0.98(\mathrm{~s}, 3 \mathrm{H}), 1.02(\mathrm{~s}, 3 \mathrm{H}), 1.09$ (s, 3H), $1.13(\mathrm{~s}, 3 \mathrm{H})$, $1.25-1.25(\mathrm{~m}, 6 \mathrm{H}), 1.95(\mathrm{~s}, 3 \mathrm{H}), 3.34\left(\mathrm{dd},{ }^{3} \mathrm{~J}=5.9 \mathrm{~Hz},{ }^{2} J=13.3 \mathrm{~Hz}, 1 \mathrm{H}\right)$, $3.62\left(\mathrm{dd},{ }^{3} J=5.9 \mathrm{~Hz},{ }^{2} J=13.3 \mathrm{~Hz}, 1 \mathrm{H}\right), 3.93(\mathrm{~s}, 3 \mathrm{H}), 6.48\left(\right.$, ${ }^{*}$, ${ }^{3} J=5.9 \mathrm{~Hz}$, 1H), 7.28-7.49 (m, 3H), $7.90\left(\mathrm{dd},{ }^{4} J=1.5 \mathrm{~Hz},{ }^{3} J=7.8 \mathrm{~Hz}, 1 \mathrm{H}\right)$.

${ }^{13} \mathrm{C}-\mathrm{NMR}: \quad\left(62.9 \mathrm{MHz}, \mathrm{CDCl}_{3}\right): \delta=17.0\left(\mathrm{CH}_{2}\right), 19.9\left(\mathrm{CH}_{3}\right), 20.2\left(\mathrm{CH}_{3}\right), 21.2\left(\mathrm{CH}_{3}\right)$, $32.7\left(\mathrm{CH}_{3}\right), 33.7\left(\mathrm{CH}_{3}\right), 37.6\left(\mathrm{CH}_{2}\right), 39.9\left(\mathrm{CH}_{2}\right), 40.1\left(\mathrm{CH}_{2}\right), 51.9\left(\mathrm{CH}_{3}\right)$, $59.0\left(\mathrm{C}_{\mathrm{q}}\right), 60.5\left(\mathrm{C}_{\mathrm{q}}\right), 99.7(\mathrm{CH}), 126.6(\mathrm{CH}), 130.3(\mathrm{CH}), 130.8\left(\mathrm{C}_{\mathrm{q}}\right), 131.3$ $(\mathrm{CH}), 133.0(\mathrm{CH}), 136.7\left(\mathrm{C}_{\mathrm{q}}\right), 167.9\left(\mathrm{C}_{\mathrm{q}}\right), 169.6\left(\mathrm{C}_{\mathrm{q}}\right)$.

ESI-MS: $\quad m / z=378\left[\mathrm{M}^{+}+\mathrm{H}\right]$.

HR-ESI-MS: $\quad \mathrm{C}_{21} \mathrm{H}_{32} \mathrm{NO}_{5}\left[\mathrm{M}^{+}+\mathrm{H}\right]$ calcd.: 378.2275 , found.: 378.2265 .

\section{3-(4-Methoxy-phenyl)-2-(2,2,6,6-tetramethyl-piperidin-1-yloxy)-propionitrile (4j)}

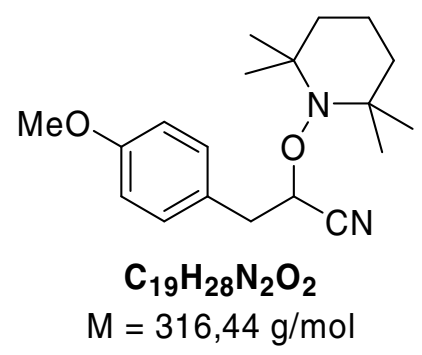

Light yellow oil.

$R_{\mathrm{f}}: \quad 0.70\left(100 \% \mathrm{CH}_{2} \mathrm{Cl}_{2}\right)$

${ }^{1} \mathrm{H}-\mathrm{NMR}: \quad\left(250 \mathrm{MHz}, \mathrm{CDCl}_{3}\right): \delta=1.09(\mathrm{~s}, 6 \mathrm{H}), 1.13(\mathrm{~s}, 3 \mathrm{H}), 1.20-1.60(\mathrm{~m}, 6 \mathrm{H}), 1.27$ $(\mathrm{s}, 3 \mathrm{H}), 3.06\left(\mathrm{dd},{ }^{3} J=7.2 \mathrm{~Hz},{ }^{2} J=13.9 \mathrm{~Hz}, 1 \mathrm{H}\right), 3.12\left(\mathrm{dd},{ }^{3} J=6.2 \mathrm{~Hz},{ }^{2} J=\right.$ $13.9 \mathrm{~Hz}, 1 \mathrm{H}), 3.80(\mathrm{~s}, 3 \mathrm{H}), 4.74\left(\mathrm{dd},{ }^{3} J=6.2 \mathrm{~Hz},{ }^{3} J=7.2 \mathrm{~Hz}, 1 \mathrm{H}\right), 6.86(\mathrm{~d}$, $\left.{ }^{3} J=8.8 \mathrm{~Hz}, 2 \mathrm{H}\right), 7.22\left(\mathrm{~d},{ }^{3} J=8.8 \mathrm{~Hz}, 2 \mathrm{H}\right)$.

${ }^{13} \mathrm{C}-\mathrm{NMR}: \quad\left(62.9 \mathrm{MHz}, \mathrm{CDCl}_{3}\right): \delta=17.0\left(\mathrm{CH}_{2}\right), 20.3\left(\mathrm{CH}_{3}\right), 20.5\left(\mathrm{CH}_{3}\right), 33.6\left(\mathrm{CH}_{3}\right)$, $34.0\left(\mathrm{CH}_{3}\right), 38.5\left(\mathrm{CH}_{2}\right), 39.9\left(\mathrm{CH}_{2}\right), 40.0\left(\mathrm{CH}_{2}\right), 55.2\left(\mathrm{CH}_{3}\right), 59.9\left(\mathrm{C}_{\mathrm{q}}\right), 61.0$ $\left(\mathrm{C}_{\mathrm{q}}\right), 75.3(\mathrm{CH}), 113.9(2 \times \mathrm{CH}), 119.3\left(\mathrm{C}_{\mathrm{q}}\right), 126.7\left(\mathrm{C}_{\mathrm{q}}\right), 130.8(2 \times \mathrm{CH}), 158.9$ $\left(\mathrm{C}_{\mathrm{q}}\right)$.

EI-MS: $\quad m / z(\%)=316(1)\left[\mathrm{M}^{+}+\mathrm{H}\right], 301(3), 160(52), 159(43), 156(100), 144(10)$, 123 (20), 121 (11), 116 (12), 89 (9), 69 (17). 
ESI-MS: $\quad m / z=317\left[\mathrm{M}^{+}+\mathrm{H}\right]$.

HR-ESI -MS: $\quad \mathrm{C}_{19} \mathrm{H}_{29} \mathrm{~N}_{2} \mathrm{O}_{2}\left[\mathrm{M}^{+}+\mathrm{H}\right] \quad$ calcd.: 317.2224, found.: 317.2216.

\section{3-(4-Fluoro-phenyl)- $N, N$-dimethyl-2-(2,2,6,6-tetramethyl-piperidin-1-yloxy)-}

propionamide $(4 k)$

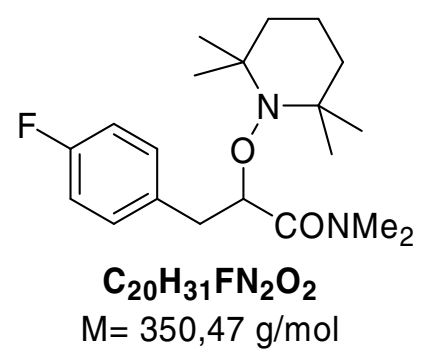

Light brown solid.

m.p.: $\quad 84-85^{\circ} \mathrm{C}$

$R_{\mathrm{f}}: \quad 0.70\left(20: 1 \mathrm{CH}_{2} \mathrm{Cl}_{2}-\mathrm{MeOH}\right)$

${ }^{1} \mathrm{H}-\mathrm{NMR}: \quad\left(360 \mathrm{MHz}, \mathrm{CDCl}_{3}\right): \delta=0.99(\mathrm{~s}, 3 \mathrm{H}), 1.11(\mathrm{~s}, 3 \mathrm{H}), 1.13(\mathrm{~s}, 3 \mathrm{H}), 1.20-1.60$ $(\mathrm{m}, 6 \mathrm{H}), 1.24(\mathrm{~s}, 3 \mathrm{H}), 2.77(\mathrm{~s}, 3 \mathrm{H}), 2.78(\mathrm{~s}, 3 \mathrm{H}), 3.06\left(\mathrm{dd},{ }^{3} \mathrm{~J}=10.1 \mathrm{~Hz},{ }^{2} \mathrm{~J}=\right.$ $13.0 \mathrm{~Hz}, 1 \mathrm{H}), 3.17\left(\mathrm{dd},{ }^{3} J=5.0 \mathrm{~Hz},{ }^{2} J=13.0 \mathrm{~Hz}, 1 \mathrm{H}\right), 4.75\left(\mathrm{dd},{ }^{3} J=5.0\right.$ $\left.\mathrm{Hz},{ }^{2} J=10.1 \mathrm{~Hz}, 1 \mathrm{H}\right), 6.92\left(\mathrm{dd},{ }^{3} J_{\mathrm{HF}}=8.6 \mathrm{~Hz},{ }^{3} J=8.6 \mathrm{~Hz}, 2 \mathrm{H}\right), 7.14(\mathrm{dd}$, $\left.{ }^{4} J_{\mathrm{HF}}=5.4 \mathrm{~Hz},{ }^{3} \mathrm{~J}=8.6 \mathrm{~Hz}, 2 \mathrm{H}\right)$.

${ }^{13} \mathrm{C}-\mathrm{NMR}: \quad\left(90.6 \mathrm{MHz}, \mathrm{CDCl}_{3}\right): \delta=16.9\left(\mathrm{CH}_{2}\right), 19.9\left(\mathrm{CH}_{3}\right), 20.2\left(\mathrm{CH}_{3}\right), 32.5\left(\mathrm{CH}_{3}\right)$, $33.3\left(\mathrm{CH}_{3}\right), 35.3\left(\mathrm{CH}_{3}\right), 37.0\left(\mathrm{CH}_{3}\right), 37.5\left(\mathrm{CH}_{2}\right), 40.1\left(\mathrm{CH}_{2}\right), 40.3\left(\mathrm{CH}_{2}\right)$, $59.2\left(\mathrm{C}_{\mathrm{q}}\right), 60.4\left(\mathrm{C}_{\mathrm{q}}\right), 80.0\left(\mathrm{~d},{ }^{5} J_{\mathrm{CF}}=1.3 \mathrm{~Hz}, \mathrm{CH}\right), 114.8\left(\mathrm{~d},{ }^{2} J_{\mathrm{CF}}=23.3 \mathrm{~Hz}\right.$, $2 \times \mathrm{CH}), 130.8\left(\mathrm{~d},{ }^{3} J_{\mathrm{CF}}=7.9 \mathrm{~Hz}, 2 \times \mathrm{CH}\right), 132.5\left(\mathrm{~d},{ }^{4} J_{\mathrm{CF}}=3.3 \mathrm{~Hz}, \mathrm{C}_{\mathrm{q}}\right), 161.4$ $\left(\mathrm{d},{ }^{1} J_{\mathrm{CF}}=244.4 \mathrm{~Hz}, \mathrm{C}_{\mathrm{q}}\right), 172.0\left(\mathrm{C}_{\mathrm{q}}\right)$.

${ }^{19}$ F-NMR: $\quad\left(235.3 \mathrm{MHz}, \mathrm{CDCl}_{3}\right): \delta=-117.1$.

ESI-MS: $\quad m / z=351\left[\mathrm{M}^{+}+\mathrm{H}\right]$.

HR-ESI-MS: $\quad \mathrm{C}_{20} \mathrm{H}_{32} \mathrm{FN}_{2} \mathrm{O}_{2}\left[\mathrm{M}^{+}+\mathrm{H}\right] \quad$ calcd.: 351.2443, found.: 351.2435.

1-[2-(4-Methoxy-phenyl)-1-phenyl-ethoxy]-2,2,6,6-tetramethyl-piperidine (4l) 


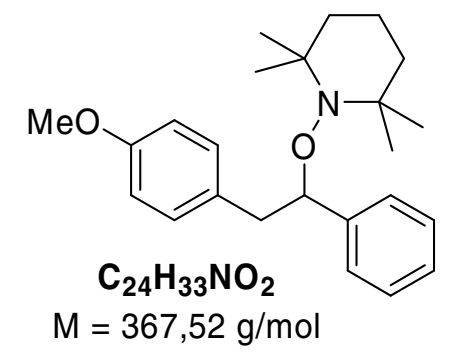

Light brown oil.

$R_{\mathrm{f}}: \quad 0.70\left(100 \% \mathrm{CH}_{2} \mathrm{Cl}_{2}\right)$

${ }^{1} \mathrm{H}-\mathrm{NMR}: \quad\left(360 \mathrm{MHz}, \mathrm{CDCl}_{3}\right): \delta=0.61(\mathrm{~s}, 3 \mathrm{H}), 1.04(\mathrm{~s}, 3 \mathrm{H}), 1.20(\mathrm{~s}, 3 \mathrm{H}), 1.20-1.60$ $(\mathrm{m}, 6 \mathrm{H}), 1.38(\mathrm{~s}, 3 \mathrm{H}), 2.85\left(\mathrm{dd},{ }^{3} J=10.1 \mathrm{~Hz},{ }^{2} J=13.0 \mathrm{~Hz}, 1 \mathrm{H}\right), 3.55\left(\mathrm{dd},{ }^{3} J\right.$ $\left.=4.3 \mathrm{~Hz},{ }^{2} J=13.0 \mathrm{~Hz}, 1 \mathrm{H}\right), 3.73(\mathrm{~s}, 3 \mathrm{H}), 4.75\left(\mathrm{dd},{ }^{3} J=4.3 \mathrm{~Hz},{ }^{3} J=10.1\right.$ $\mathrm{Hz}, 1 \mathrm{H}), 6.66\left(\mathrm{~d},{ }^{3} \mathrm{~J}=8.6 \mathrm{~Hz}, 2 \mathrm{H}\right), 7.22\left(\mathrm{~d},{ }^{3} \mathrm{~J}=8.6 \mathrm{~Hz}, 2 \mathrm{H}\right), 7.07-7.10(\mathrm{~m}$, $2 \mathrm{H}), 7.16-7.22(\mathrm{~m}, 3 \mathrm{H})$.

${ }^{13} \mathrm{C}-\mathrm{NMR}: \quad\left(90.6 \mathrm{MHz}, \mathrm{CDCl}_{3}\right): \delta=17.0\left(\mathrm{CH}_{2}\right), 20.4\left(\mathrm{br}, 2 \times \mathrm{CH}_{3}\right), 34.3\left(\mathrm{CH}_{3}\right), 34.4$ $\left(\mathrm{CH}_{3}\right), 40.5\left(2 \times \mathrm{CH}_{2}\right), 42.1\left(\mathrm{CH}_{2}\right), 55.1\left(\mathrm{CH}_{3}\right), 59.8\left(\right.$ br, $\left.2 \times \mathrm{C}_{\mathrm{q}}\right), 88.8(\mathrm{CH})$, $113.3(2 \times \mathrm{CH}), 126.9(\mathrm{CH}), 127.5(2 \times \mathrm{CH}), 128.1(2 \times \mathrm{CH}), 130.5\left(\mathrm{C}_{\mathrm{q}}\right), 130.6$ $(2 \times \mathrm{CH}), 142.8\left(\mathrm{C}_{\mathrm{q}}\right), 157.7\left(\mathrm{C}_{\mathrm{q}}\right)$.

ESI-MS: $\quad m / z=368\left[\mathrm{M}^{+}+\mathrm{H}\right]$.

HR-ESI -MS: $\quad \mathrm{C}_{24} \mathrm{H}_{34} \mathrm{NO}_{2}\left[\mathrm{M}^{+}+\mathrm{H}\right] \quad$ calcd.: 368.2584, found.: 368.2574.

trans-4-(4-Methoxy-phenyl)-5-(2,2,6,6-tetramethyl-piperidin-1-yloxy)-[1,3]dioxolan-2one $(\mathbf{4 m})$

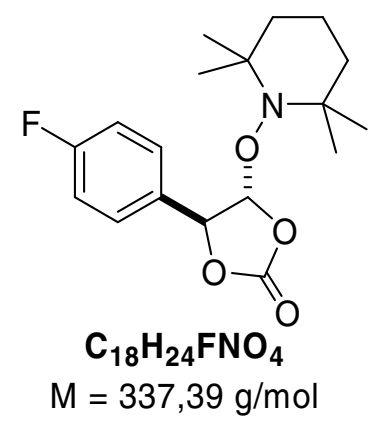

Colorless oil.

$R_{\mathrm{f}}$ :

$0.70\left(100 \% \mathrm{CH}_{2} \mathrm{Cl}_{2}\right)$ 
${ }^{1} \mathrm{H}-\mathrm{NMR}: \quad\left(360 \mathrm{MHz}, \mathrm{CDCl}_{3}\right): \delta=0.87(\mathrm{~s}, 3 \mathrm{H}), 1.13(\mathrm{~s}, 6 \mathrm{H}), 1.20-1.60(\mathrm{~m}, 6 \mathrm{H}), 1.27$ (s, $3 \mathrm{H}), 3.06\left(\mathrm{~d},{ }^{3} J=3.2 \mathrm{~Hz}, 1 \mathrm{H}\right), 5.79\left(\mathrm{~d},{ }^{3} J=3.2 \mathrm{~Hz}, 1 \mathrm{H}\right), 7.12\left(\mathrm{dd},{ }^{3} J_{\mathrm{HF}}=\right.$ $\left.8.6 \mathrm{~Hz},{ }^{3} \mathrm{~J}=8.6 \mathrm{~Hz}, 2 \mathrm{H}\right), 7.32\left(\mathrm{~d},{ }^{4} J_{\mathrm{HF}}=5.0 \mathrm{~Hz},{ }^{3} J=8.6 \mathrm{~Hz}, 2 \mathrm{H}\right)$.

${ }^{13} \mathrm{C}-\mathrm{NMR}: \quad\left(90.6 \mathrm{MHz}, \mathrm{CDCl}_{3}\right): \delta=16.8\left(\mathrm{CH}_{2}\right), 20.2\left(\mathrm{CH}_{3}\right), 20.4\left(\mathrm{CH}_{3}\right), 32.9\left(\mathrm{CH}_{3}\right)$, $33.7\left(\mathrm{CH}_{3}\right), 39.6\left(\mathrm{CH}_{2}\right), 40.1\left(\mathrm{CH}_{2}\right), 59.8\left(\mathrm{C}_{\mathrm{q}}\right), 60.9\left(\mathrm{C}_{\mathrm{q}}\right), 82.6(\mathrm{CH}), 110.4$ $(\mathrm{CH}), 116.3\left(\mathrm{~d},{ }^{2} J_{\mathrm{CF}}=23.3 \mathrm{~Hz}, 2 \times \mathrm{CH}\right), 128.4\left(\mathrm{~d},{ }^{3} J_{\mathrm{CF}}=8.7 \mathrm{~Hz}, 2 \times \mathrm{CH}\right)$, $130.7\left(\mathrm{~d},{ }^{4} J_{\mathrm{CF}}=3.4 \mathrm{~Hz}, \mathrm{C}_{\mathrm{q}}\right), 153.1\left(\mathrm{C}_{\mathrm{q}}\right), 163.4\left(\mathrm{~d},{ }^{1} J_{\mathrm{CF}}=250.1 \mathrm{~Hz}, \mathrm{C}_{\mathrm{q}}\right)$.

${ }^{19}$ F-NMR: $\quad\left(235.3 \mathrm{MHz}, \mathrm{CDCl}_{3}\right): \delta=-111.1$.

ESI-MS: $\quad m / z=338\left[\mathrm{M}^{+}+\mathrm{H}\right]$.

HR-ESI-MS: $\quad \mathrm{C}_{18} \mathrm{H}_{25} \mathrm{FNO}_{4}\left[\mathrm{M}^{+}+\mathrm{H}\right]$ calcd.: 338.1762 , found.: 338.1758 .

cis-4-(4-Methoxy-phenyl)-5-(2,2,6,6-tetramethyl-piperidin-1-yloxy)-[1,3]dioxolan-2-one (4m')

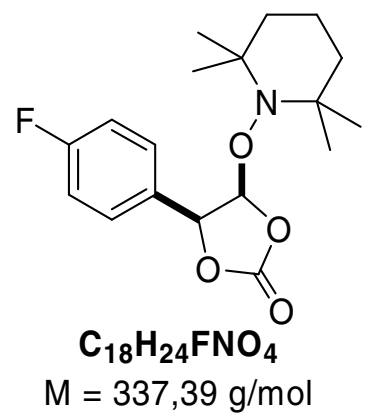

Colorless oil.

$R_{\mathrm{f}}: \quad 0.45\left(100 \% \mathrm{CH}_{2} \mathrm{Cl}_{2}\right)$

${ }^{1} \mathrm{H}-\mathrm{NMR}: \quad\left(250 \mathrm{MHz}, \mathrm{CDCl}_{3}\right): \delta=0.42(\mathrm{~s}, 3 \mathrm{H}), 0.82(\mathrm{~s}, 3 \mathrm{H}), 0.97(\mathrm{~s}, 3 \mathrm{H}) 1.20-1.60$ $(\mathrm{m}, 6 \mathrm{H}), 1.25(\mathrm{~s}, 3 \mathrm{H}), 5.65\left(\mathrm{~d},{ }^{3} J=6.3 \mathrm{~Hz}, 1 \mathrm{H}\right), 6.02\left(\mathrm{~d},{ }^{3} J=6.3 \mathrm{~Hz}, 1 \mathrm{H}\right)$, $7.11\left(\mathrm{dd},{ }^{3} J_{\mathrm{HF}}=8.8 \mathrm{~Hz},{ }^{3} J=8.8 \mathrm{~Hz}, 2 \mathrm{H}\right), 7.40\left(\mathrm{~d},{ }^{4} J_{\mathrm{HF}}=5.3 \mathrm{~Hz},{ }^{3} J=8.8 \mathrm{~Hz}\right.$, $2 \mathrm{H})$.

${ }^{13} \mathrm{C}-\mathrm{NMR}: \quad\left(90.6 \mathrm{MHz}, \mathrm{CDCl}_{3}\right): \delta=16.8\left(\mathrm{CH}_{2}\right), 19.6\left(\mathrm{CH}_{3}\right), 20.7\left(\mathrm{CH}_{3}\right), 32.3\left(\mathrm{CH}_{3}\right)$, $34.0\left(\mathrm{CH}_{3}\right), 39.6\left(\mathrm{CH}_{2}\right), 40.0\left(\mathrm{CH}_{2}\right), 59.7\left(\mathrm{C}_{\mathrm{q}}\right), 61.1\left(\mathrm{C}_{\mathrm{q}}\right), 81.1(\mathrm{CH}), 104.7$ $(\mathrm{CH}), 116.3\left(\mathrm{~d},{ }^{2} J_{\mathrm{CF}}=22.0 \mathrm{~Hz}, 2 \times \mathrm{CH}\right), 127.6\left(\mathrm{~d},{ }^{4} J_{\mathrm{CF}}=3.4 \mathrm{~Hz}, \mathrm{C}_{\mathrm{q}}\right), 130.0$ $\left(\mathrm{d},{ }^{3} J_{\mathrm{CF}}=8.5 \mathrm{~Hz}, 2 \times \mathrm{CH}\right), 153.5\left(\mathrm{C}_{\mathrm{q}}\right), 163.4\left(\mathrm{~d},{ }^{1} J_{\mathrm{CF}}=249.0 \mathrm{~Hz}, \mathrm{C}_{\mathrm{q}}\right)$.

${ }^{19}$ F-NMR: $\quad\left(235.3 \mathrm{MHz}, \mathrm{CDCl}_{3}\right): \delta=-111.9$. 

acid methyl ester (4n)

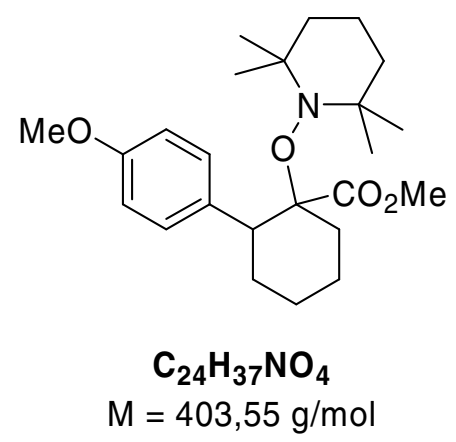

Colorless solid.

m.p. $\quad 123-124^{\circ} \mathrm{C}$

$R_{\mathrm{f}}: \quad 0.50\left(1: 1 \mathrm{CH}_{2} \mathrm{Cl}_{2}\right.$-pentane $)$

${ }^{1} \mathrm{H}-\mathrm{NMR}: \quad\left(360 \mathrm{MHz}, \mathrm{CDCl}_{3}\right): \delta=0.85(\mathrm{~s}, 3 \mathrm{H}), 0.99(\mathrm{~s}, 3 \mathrm{H}), 1.22(\mathrm{~s}, 3 \mathrm{H}), 1.25-1.60$ $(\mathrm{m}, 8 \mathrm{H}), 1.37(\mathrm{~s}, 3 \mathrm{H}), 1.62-1.70(\mathrm{~m}, 1 \mathrm{H}), 1.74-1.98(\mathrm{~m}, 4 \mathrm{H}), 2.17-2.32$ $(\mathrm{m}, 2 \mathrm{H}), 3.09\left(\mathrm{dd},{ }^{3} J=3.2 \mathrm{~Hz},{ }^{2} J=13.0 \mathrm{~Hz}, 1 \mathrm{H}\right), 3.41(\mathrm{~s}, 3 \mathrm{H}), 3.80(\mathrm{~s}, 3 \mathrm{H})$, $6.78\left(\mathrm{~d},{ }^{3} J=8.8 \mathrm{~Hz}, 2 \mathrm{H}\right), 7.09\left(\mathrm{~d},{ }^{3} J=8.8 \mathrm{~Hz}, 2 \mathrm{H}\right)$.

${ }^{13} \mathrm{C}-\mathrm{NMR}: \quad\left(90.6 \mathrm{MHz}, \mathrm{CDCl}_{3}\right): \delta=16.9\left(\mathrm{CH}_{2}\right), 20.2\left(\mathrm{CH}_{3}\right), 21.2\left(\mathrm{CH}_{3}\right), 24.1\left(\mathrm{CH}_{2}\right)$, $26.1\left(\mathrm{CH}_{2}\right), 30.0\left(\mathrm{CH}_{2}\right), 32.1\left(\mathrm{CH}_{2}\right), 33.0\left(\mathrm{CH}_{3}\right), 34.6\left(\mathrm{CH}_{3}\right), 40.9\left(\mathrm{CH}_{2}\right)$, $41.5\left(\mathrm{CH}_{2}\right), 50.1\left(\mathrm{CH}_{3}\right), 51.3(\mathrm{CH}), 55.1\left(\mathrm{CH}_{3}\right), 59.9\left(\mathrm{C}_{\mathrm{q}}\right), 60.0\left(\mathrm{C}_{\mathrm{q}}\right), 85.5$ $\left(\mathrm{C}_{\mathrm{q}}\right), 112.3(2 \times \mathrm{CH}), 129.8(2 \times \mathrm{CH}), 134.7\left(\mathrm{C}_{\mathrm{q}}\right), 158.0\left(\mathrm{C}_{\mathrm{q}}\right), 174.4\left(\mathrm{C}_{\mathrm{q}}\right)$.

ESI-MS: $\quad m / z=404\left[\mathrm{M}^{+}+\mathrm{H}\right]$.

HR-ESI -MS: $\quad \mathrm{C}_{24} \mathrm{H}_{38} \mathrm{NO}_{4}\left[\mathrm{M}^{+}+\mathrm{H}\right] \quad$ calcd.: 404.2796, found.: 404.2787. 


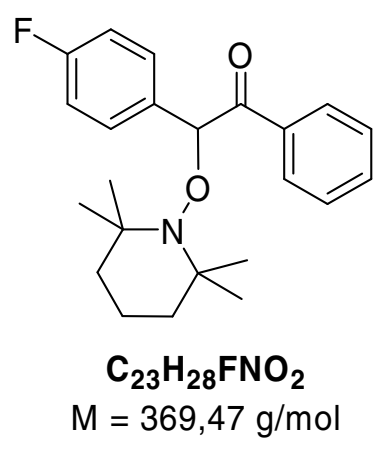

Light brown oil.

$R_{\mathrm{f}}$ :

0.50 (1:20 EtOAc-pentane)

${ }^{1} \mathrm{H}-\mathrm{NMR}: \quad\left(250 \mathrm{MHz}, \mathrm{CDCl}_{3}\right): \delta=0.79(\mathrm{~s}, 3 \mathrm{H}), 0.99(\mathrm{~s}, 3 \mathrm{H}), 1.17(\mathrm{~s}, 6 \mathrm{H}), 1.20-1.60$ $(\mathrm{m}, 6 \mathrm{H}), 5.99(\mathrm{~s}, 1 \mathrm{H}), 6.97\left(\mathrm{dd},{ }^{3} J_{\mathrm{HF}}=8.8 \mathrm{~Hz},{ }^{3} J=8.8 \mathrm{~Hz}, 2 \mathrm{H}\right), 7.38-7.51$ $(\mathrm{m}, 5 \mathrm{H}), 8.05\left(\mathrm{dd},{ }^{4} J_{\mathrm{HF}}=2.3 \mathrm{~Hz},{ }^{3} J=8.8 \mathrm{~Hz}, 2 \mathrm{H}\right)$.

${ }^{13} \mathrm{C}-\mathrm{NMR}: \quad\left(90.6 \mathrm{MHz}, \mathrm{CDCl}_{3}\right): \delta=16.9\left(\mathrm{CH}_{2}\right), 20.1\left(\mathrm{CH}_{3}\right), 20.2\left(\mathrm{CH}_{3}\right), 33.2\left(\mathrm{CH}_{3}\right)$, $33.6\left(\mathrm{CH}_{3}\right), 40.1\left(2 \times \mathrm{CH}_{2}\right), 59.6\left(\mathrm{C}_{\mathrm{q}}\right), 60.0\left(\mathrm{C}_{\mathrm{q}}\right), 92.0(\mathrm{CH}), 115.2\left(\mathrm{~d},{ }^{2} J_{\mathrm{CF}}=\right.$ $21.6 \mathrm{~Hz}, 2 \times \mathrm{CH}), 128.3(2 \times \mathrm{CH}), 128.8\left(\mathrm{~d},{ }^{3} J_{\mathrm{CF}}=8.1 \mathrm{~Hz}, 2 \times \mathrm{CH}\right), 129.0$ $(2 \times \mathrm{CH}), 132.9\left(\mathrm{C}_{\mathrm{q}}\right), 133.5\left(\mathrm{~d},{ }^{4} J_{\mathrm{CF}}=3.3 \mathrm{~Hz}, \mathrm{C}_{\mathrm{q}}\right), 135.0\left(\mathrm{C}_{\mathrm{q}}\right), 162.0\left(\mathrm{~d},{ }^{1} J_{\mathrm{CF}}=\right.$ $\left.246.7 \mathrm{~Hz}, \mathrm{C}_{\mathrm{q}}\right), 198.1\left(\mathrm{C}_{\mathrm{q}}\right)$.

${ }^{19}$ F-NMR: $\quad\left(235.3 \mathrm{MHz}, \mathrm{CDCl}_{3}\right): \delta=-115.0$.

ESI-MS: $\quad m / z=370\left[\mathrm{M}^{+}+\mathrm{H}\right]$.

Selected NOESY interactions for compound 5:

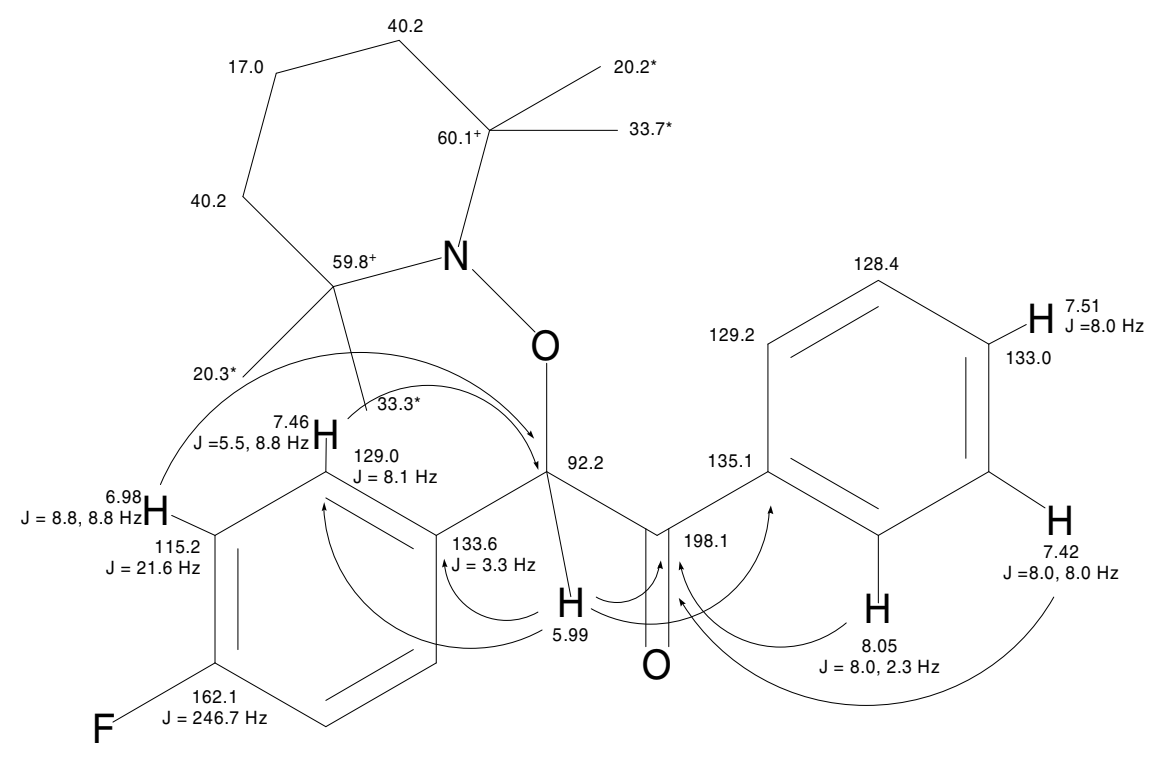


For NMR data of a related compound, see: Tetrahedron Lett. 1983, 4075. 


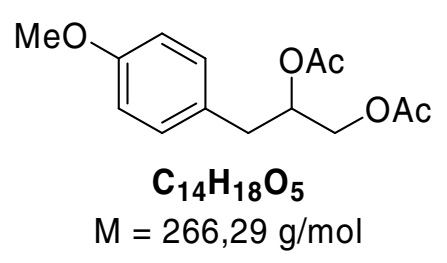

Compound $\mathbf{6}$ was prepared from $\mathbf{4 b}(0.20 \mathrm{mmol})$ by treatment with $\mathrm{Zn}$ powder $(500 \mathrm{mg})$ in $\mathrm{AcOH}(5 \mathrm{~mL})$ and $\mathrm{Ac}_{2} \mathrm{O}(3 \mathrm{~mL})$ at $100{ }^{\circ} \mathrm{C}$ for $2 \mathrm{~h}$.

\section{Colorless oil.}

$R_{\mathrm{f}}: \quad 0.65(1: 2$ EtOAc-pentane $)$

${ }^{1} \mathrm{H}-\mathrm{NMR}: \quad\left(250 \mathrm{MHz}, \mathrm{CDCl}_{3}\right): \delta=2.02(\mathrm{~s}, 3 \mathrm{H}), 2.07(\mathrm{~s}, 3 \mathrm{H}), 2.81\left(\mathrm{dd},{ }^{3} J=7.0 \mathrm{~Hz},{ }^{2} \mathrm{~J}=\right.$ $14.0 \mathrm{~Hz}, 1 \mathrm{H}), 2.88\left(\mathrm{dd},{ }^{3} J=6.8 \mathrm{~Hz},{ }^{2} J=14.0 \mathrm{~Hz}, 1 \mathrm{H}\right), 3.78(\mathrm{~s}, 3 \mathrm{H}), 3.98$ $\left(\mathrm{dd},{ }^{3} J=6.3 \mathrm{~Hz},{ }^{3} J=11.8 \mathrm{~Hz}, 1 \mathrm{H}\right), 4.22\left(\mathrm{dd},{ }^{3} J=3.8 \mathrm{~Hz},{ }^{3} J=11.8 \mathrm{~Hz}, 1 \mathrm{H}\right)$, $5.16-5.26(\mathrm{~m}, 1 \mathrm{H}), 6.82\left(\mathrm{~d},{ }^{3} J=8.8 \mathrm{~Hz}, 2 \mathrm{H}\right), 7.11\left(\mathrm{~d},{ }^{3} J=8.8 \mathrm{~Hz}, 2 \mathrm{H}\right)$.

${ }^{13} \mathrm{C}-\mathrm{NMR}: \quad\left(62.9 \mathrm{MHz}, \mathrm{CDCl}_{3}\right): \delta=20.8\left(\mathrm{CH}_{3}\right), 21.0\left(\mathrm{CH}_{3}\right), 36.1\left(\mathrm{CH}_{2}\right), 55.2\left(\mathrm{CH}_{3}\right)$, $64.2\left(\mathrm{CH}_{2}\right), 72.2(\mathrm{CH}), 113.9(2 \times \mathrm{CH}), 128.2\left(\mathrm{C}_{\mathrm{q}}\right), 130.2(2 \times \mathrm{CH}), 158.5$ $\left(\mathrm{C}_{\mathrm{q}}\right), 170.3\left(\mathrm{C}_{\mathrm{q}}\right), 170.7\left(\mathrm{C}_{\mathrm{q}}\right)$.

EI-MS: $\quad m / z(\%)=266(1)\left[\mathrm{M}^{+}\right], 206(38), 165(9), 164(25), 163(33), 147(14), 135$ (9), 121 (100), 108 (9), 91 (9).

HR-EI -MS: $\quad \mathrm{C}_{14} \mathrm{H}_{18} \mathrm{O}_{5}\left[\mathrm{M}^{+}\right] \quad$ calcd.: 266.1154, found.: 266.1148.

\section{1-Oxo-isochroman-3-carboxylic acid ethyl ester (7)}

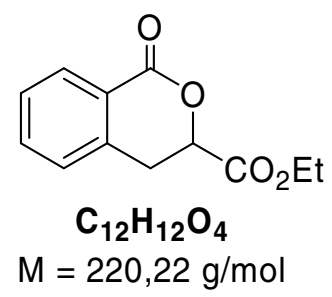

Compound 7 was prepared from $\mathbf{4 g}(0.25 \mathrm{mmol})$ by treatment with $\mathrm{Zn}$ powder $(500 \mathrm{mg})$ in $\mathrm{AcOH}(5 \mathrm{~mL})$ at $100^{\circ} \mathrm{C}$ for $2 \mathrm{~h}$.

Colorless solid.

m.p.: $\quad 86-87^{\circ} \mathrm{C}$ 
$R_{\mathrm{f}}:$

0.40 (1:3 EtOAc-pentane)

${ }^{1} \mathrm{H}-\mathrm{NMR}: \quad\left(250 \mathrm{MHz}, \mathrm{CDCl}_{3}\right): \delta=1.16\left(\mathrm{t},{ }^{3} J=7.1 \mathrm{~Hz}, 3 \mathrm{H}\right), 3.27\left(\mathrm{dd},{ }^{3} J=5.4 \mathrm{~Hz},{ }^{2} J=\right.$ $16.5 \mathrm{~Hz}, 1 \mathrm{H}), 3.44\left(\mathrm{dd},{ }^{3} J=5.4 \mathrm{~Hz},{ }^{2} J=16.5 \mathrm{~Hz}, 1 \mathrm{H}\right), 4.16\left(\mathrm{dd},{ }^{3} J=2.5\right.$ $\left.\mathrm{Hz},{ }^{3} J=7.1 \mathrm{~Hz}, 2 \mathrm{H}\right), 5.14\left(\mathrm{t},{ }^{3} J=5.4 \mathrm{~Hz}, 1 \mathrm{H}\right), 7.24\left(\mathrm{~d},{ }^{3} J=7.5 \mathrm{~Hz}, 1 \mathrm{H}\right)$, $7.39\left(\mathrm{t},{ }^{3} J=7.5 \mathrm{~Hz}, 1 \mathrm{H}\right), 7.53\left(\mathrm{dt},{ }^{4} J=1.3 \mathrm{~Hz},{ }^{3} J=7.5 \mathrm{~Hz}, 1 \mathrm{H}\right), 8.08\left(\mathrm{~d},{ }^{3} J=\right.$ $7.5 \mathrm{~Hz}, 1 \mathrm{H})$.

${ }^{13}$ C-NMR: $\quad\left(90.6 \mathrm{MHz}, \mathrm{CDCl}_{3}\right): \delta=13.9\left(\mathrm{CH}_{3}\right), 30.2\left(\mathrm{CH}_{2}\right), 62.0\left(\mathrm{CH}_{2}\right), 74.9(\mathrm{CH})$, $124.9\left(\mathrm{C}_{\mathrm{q}}\right), 127.4(\mathrm{CH}), 128.1(\mathrm{CH}), 130.1(\mathrm{CH}), 133.9(\mathrm{CH}), 136.2\left(\mathrm{C}_{\mathrm{q}}\right)$, $163.4\left(\mathrm{C}_{\mathrm{q}}\right), 168.9\left(\mathrm{C}_{\mathrm{q}}\right)$.

EI-MS: $\quad m / z(\%)=220(12)\left[\mathrm{M}^{+}\right], 148(9), 147(100), 120(6), 119(83), 91(55)$.

HR-EI-MS: $\quad \mathrm{C}_{12} \mathrm{H}_{12} \mathrm{O}_{4}\left[\mathrm{M}^{+}\right] \quad$ calcd.: 220.0736, found.: 220.2736. 
3-(4-Methoxy-phenyl)-2-(2,2,6,6-tetramethyl-piperidin-1-yloxy)-propionic acid ethyl ester (4a)

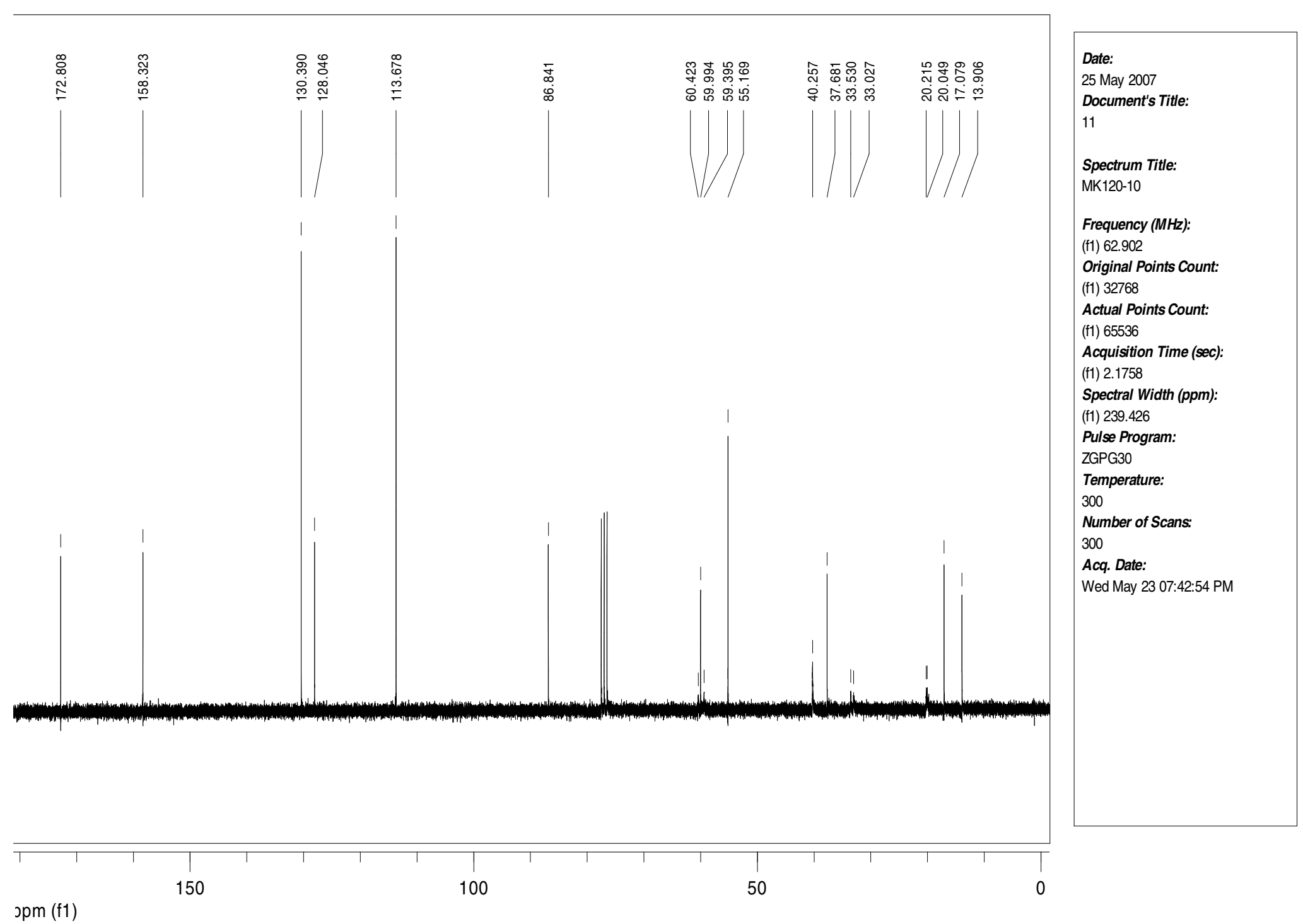


Acetic acid 3-(4-methoxy-phenyl)-2-(2,2,6,6-tetramethyl-piperidin-1-yloxy)-propyl ester (4b)

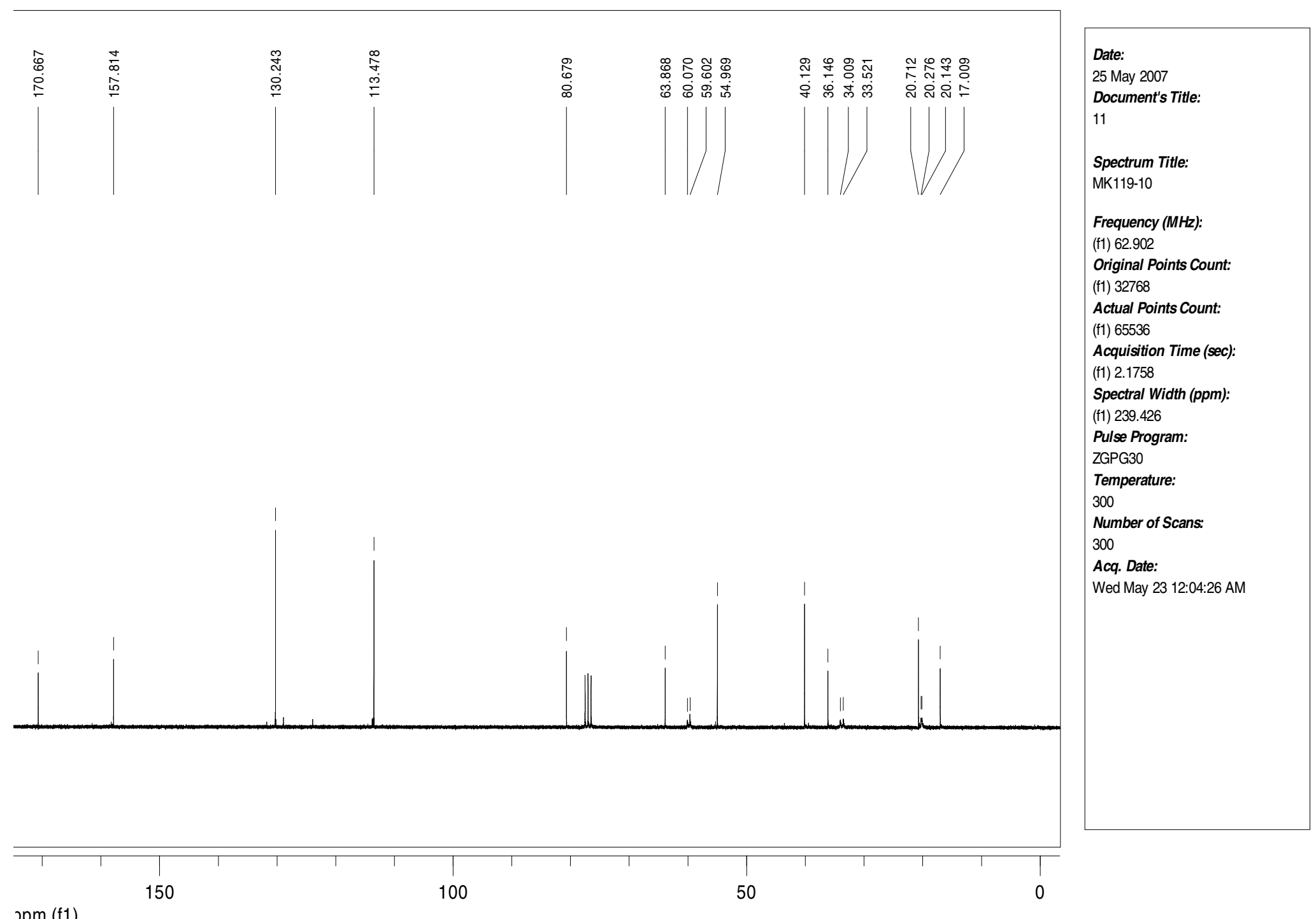


Acetic acid 2-(4-methoxy-phenyl)-1-(2,2,6,6-tetramethyl-piperidin-1-yloxy)-ethyl ester (4c)

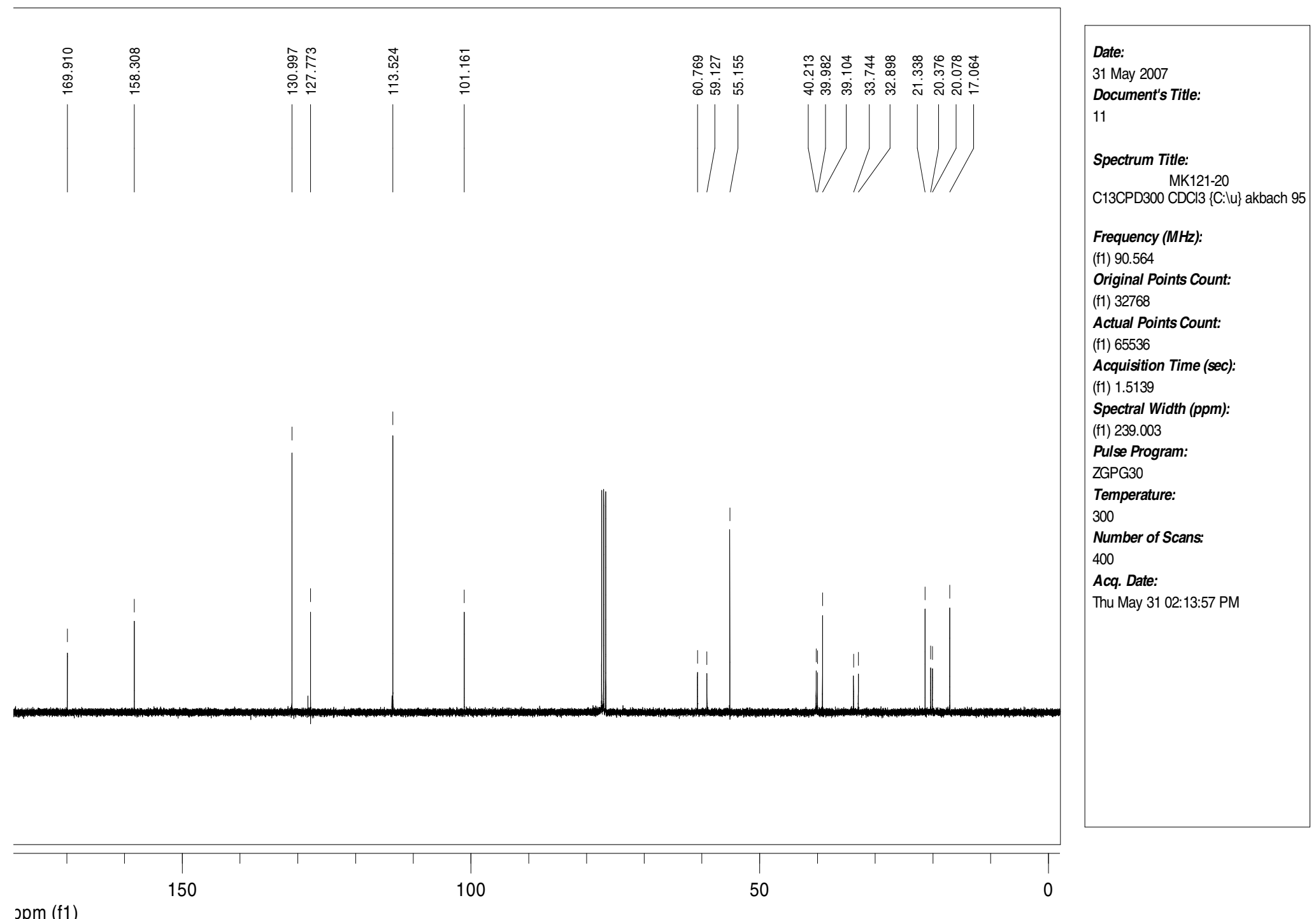


3-(4-Fluoro-phenyl)-2-(2,2,6,6-tetramethyl-piperidin-1-yloxy)-propionic acid ethyl ester (4d)

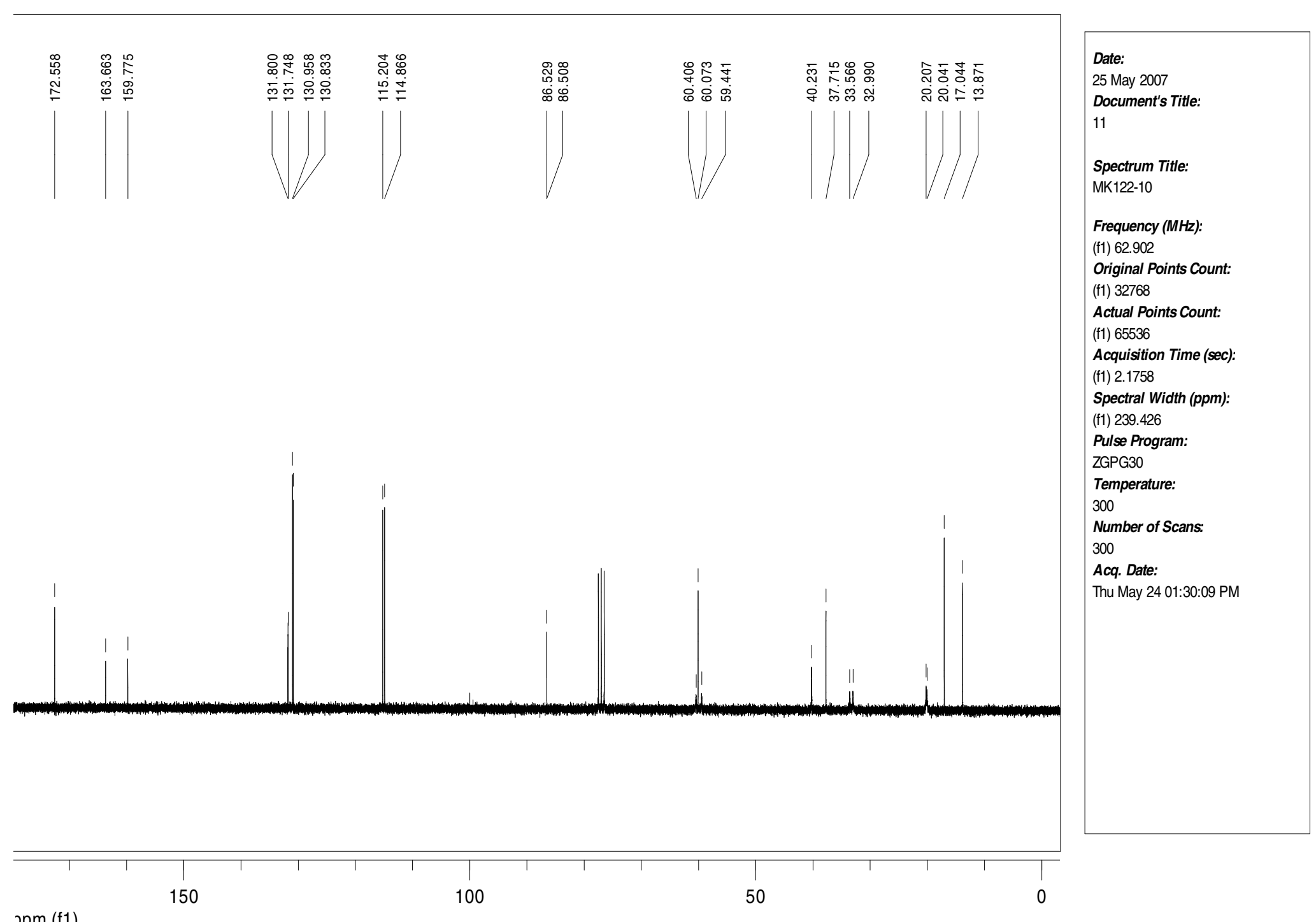


Acetic acid 3-(4-fluoro-phenyl)-2-(2,2,6,6-tetramethyl-piperidin-1-yloxy)-propyl ester (4e)

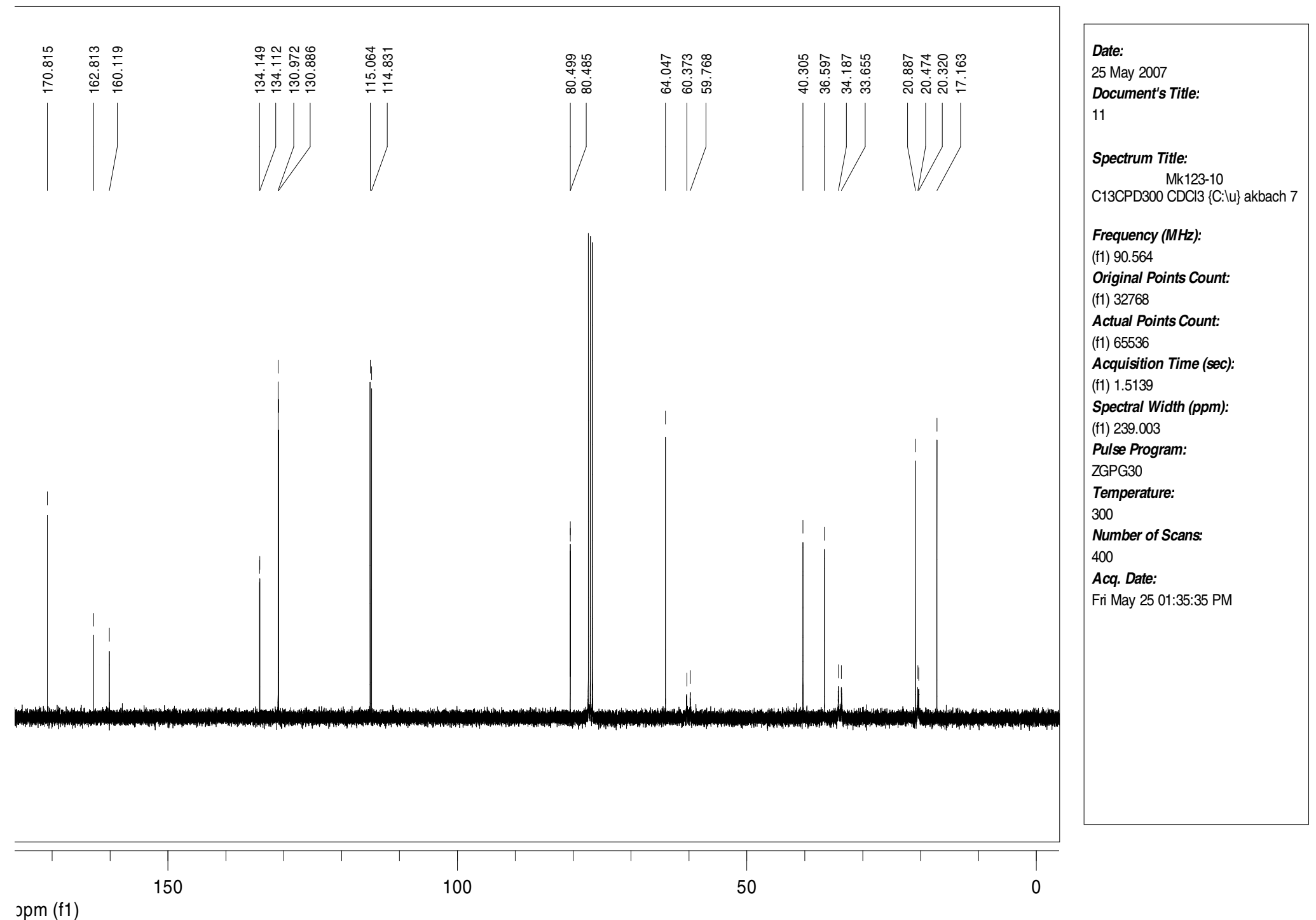


Acetic acid 2-(4-fluoro-phenyl)-1-(2,2,6,6-tetramethyl-piperidin-1-yloxy)-ethyl ester (4f)

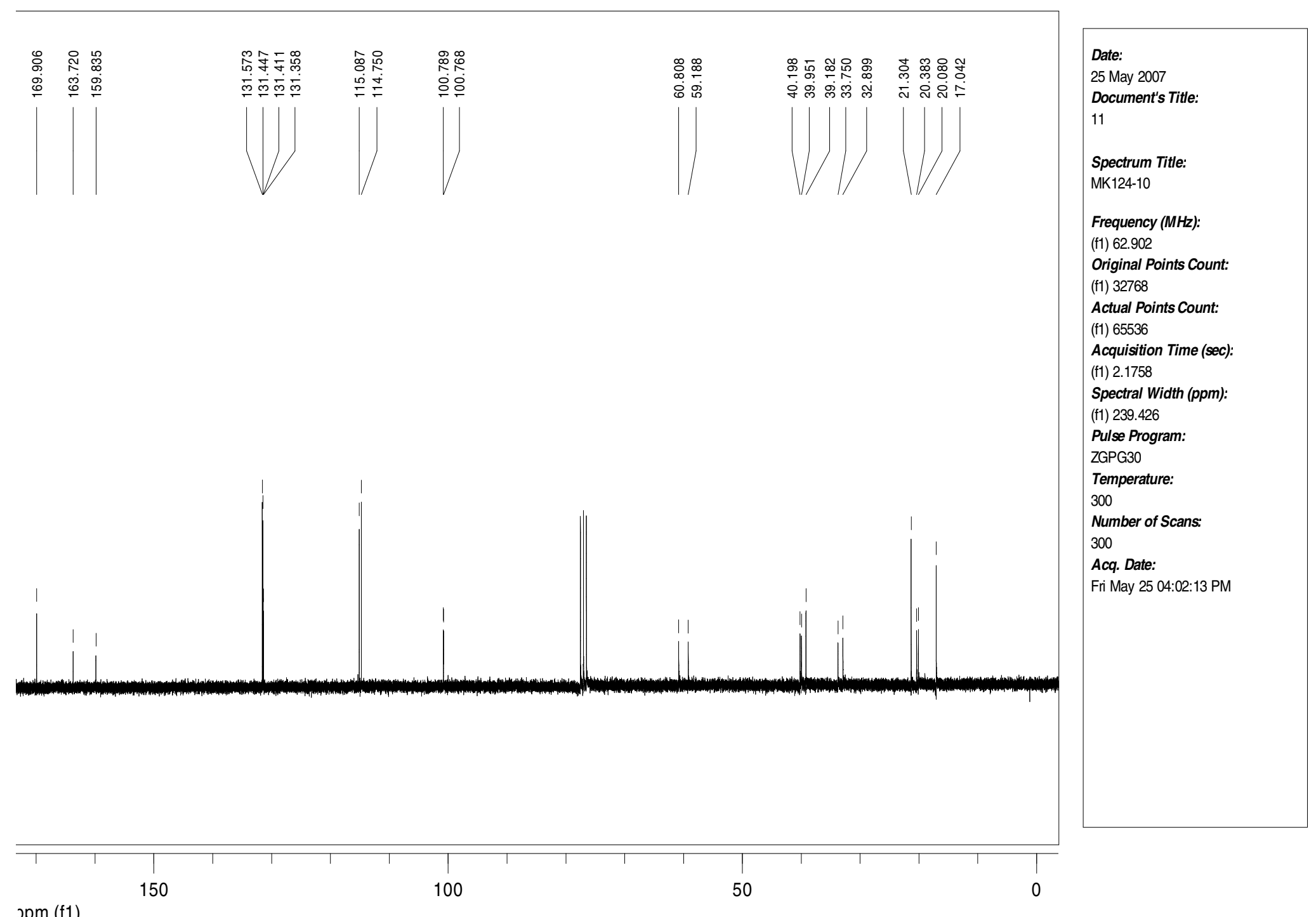


2-[2-Ethoxycarbonyl-2-(2,2,6,6-tetramethyl-piperidin-1-yloxy)-ethyl]-benzoic acid methyl ester (4g)

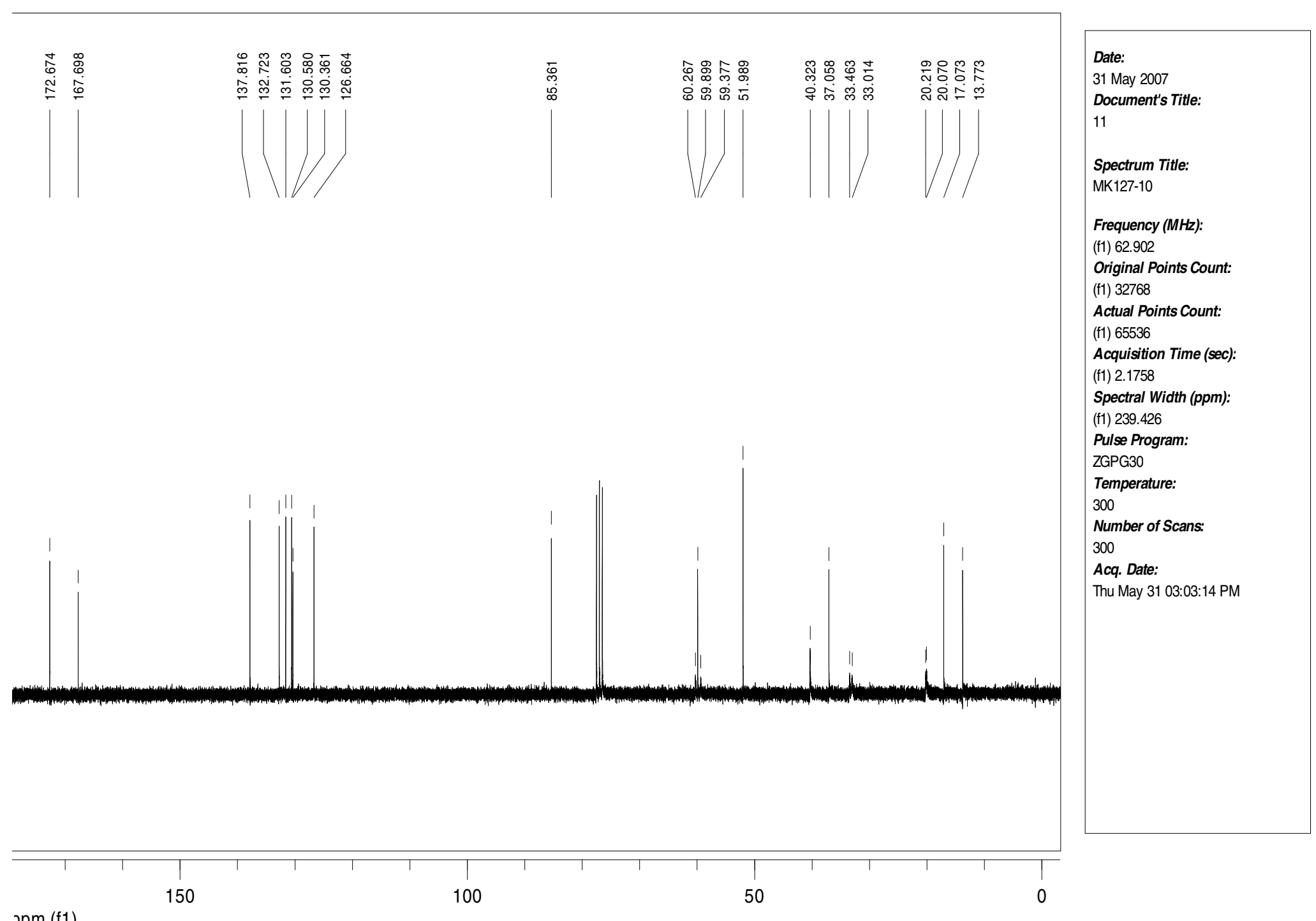


2-[3-Acetoxy-2-(2,2,6,6-tetramethyl-piperidin-1-yloxy)-propyl]-benzoic acid methyl ester (4h)

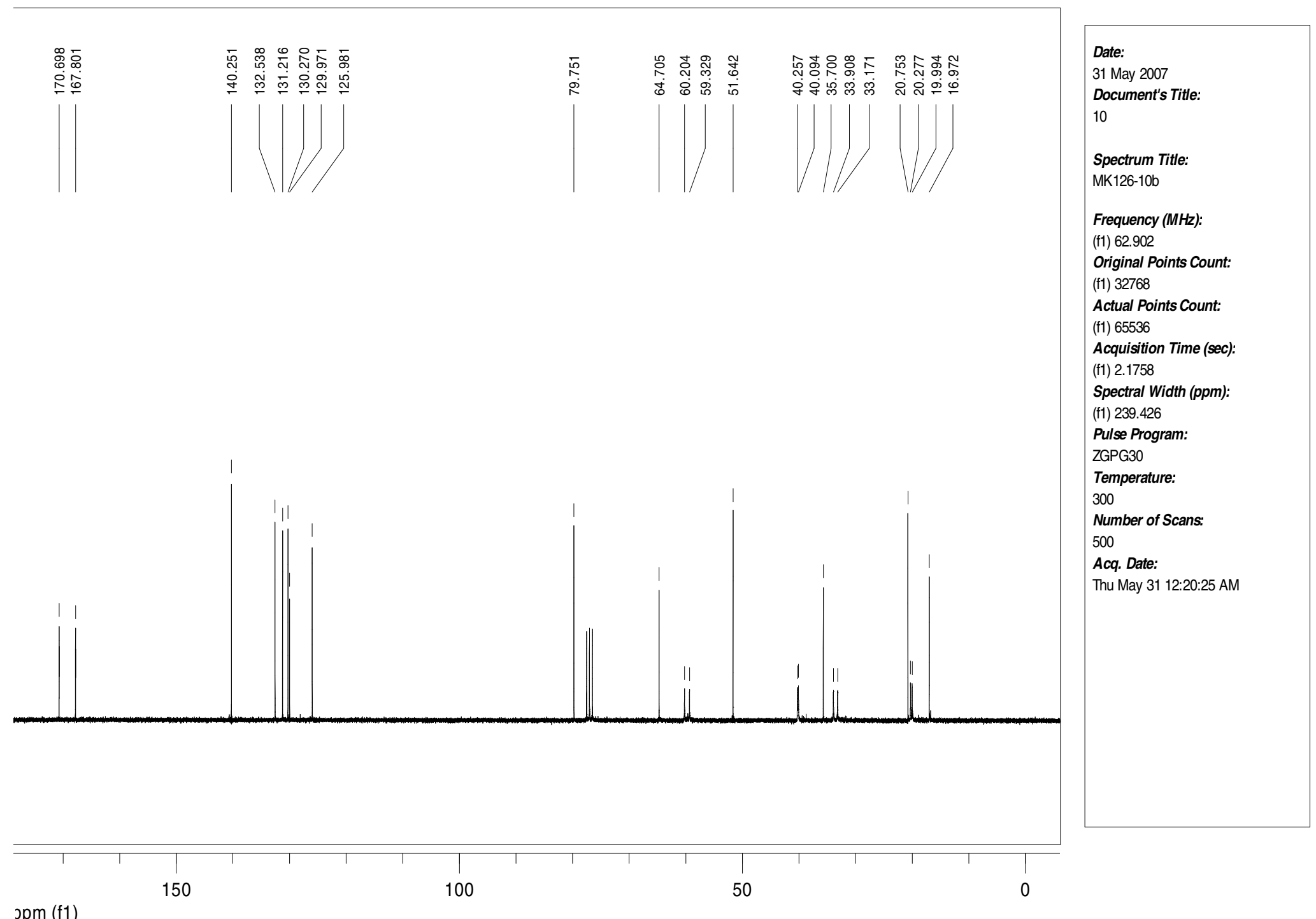


2-[2-Acetoxy-2-(2,2,6,6-tetramethyl-piperidin-1-yloxy)-ethyl]-benzoic acid methyl ester (4i)

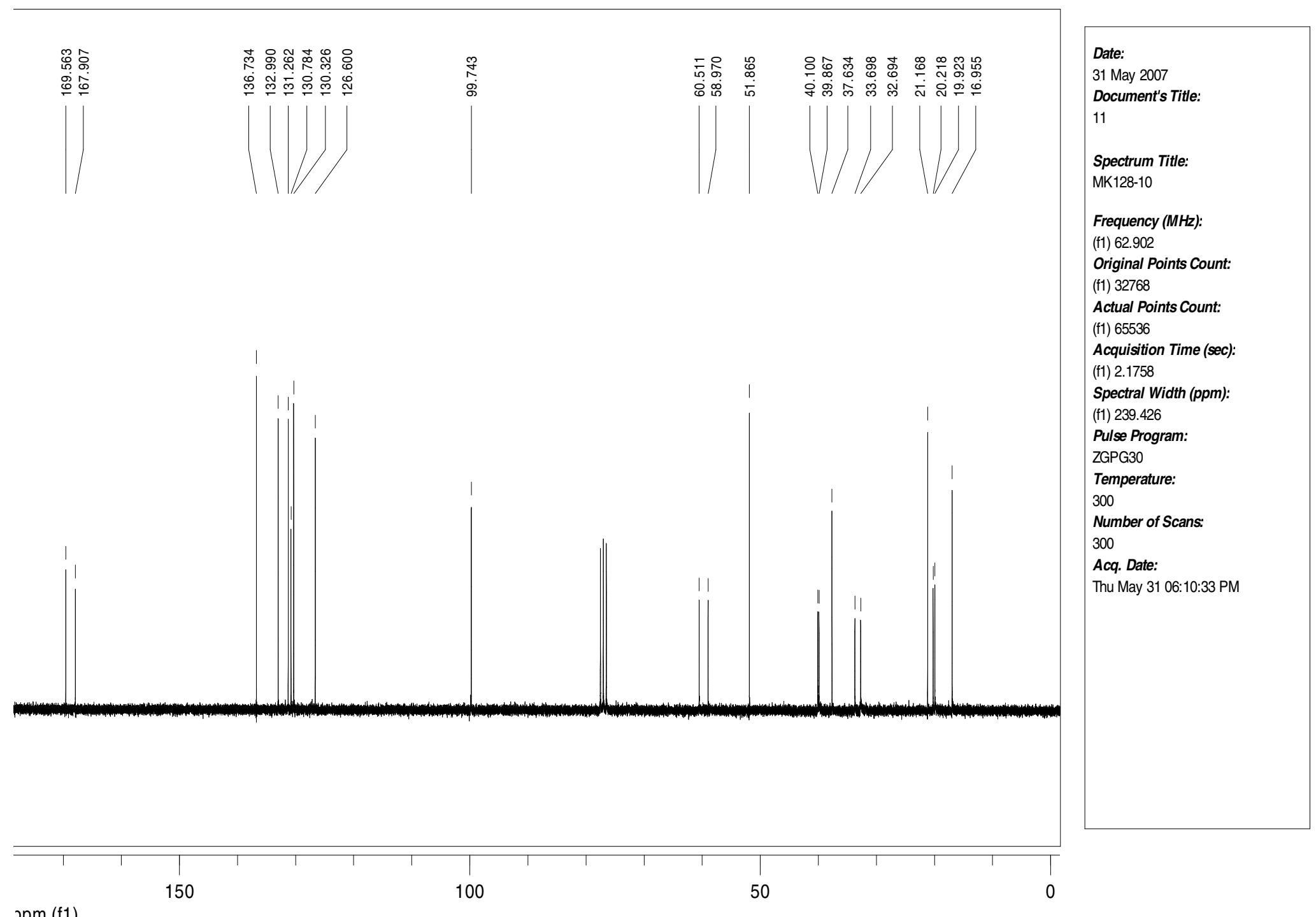


3-(4-Methoxy-phenyl)-2-(2,2,6,6-tetramethyl-piperidin-1-yloxy)-propionitrile (4j)

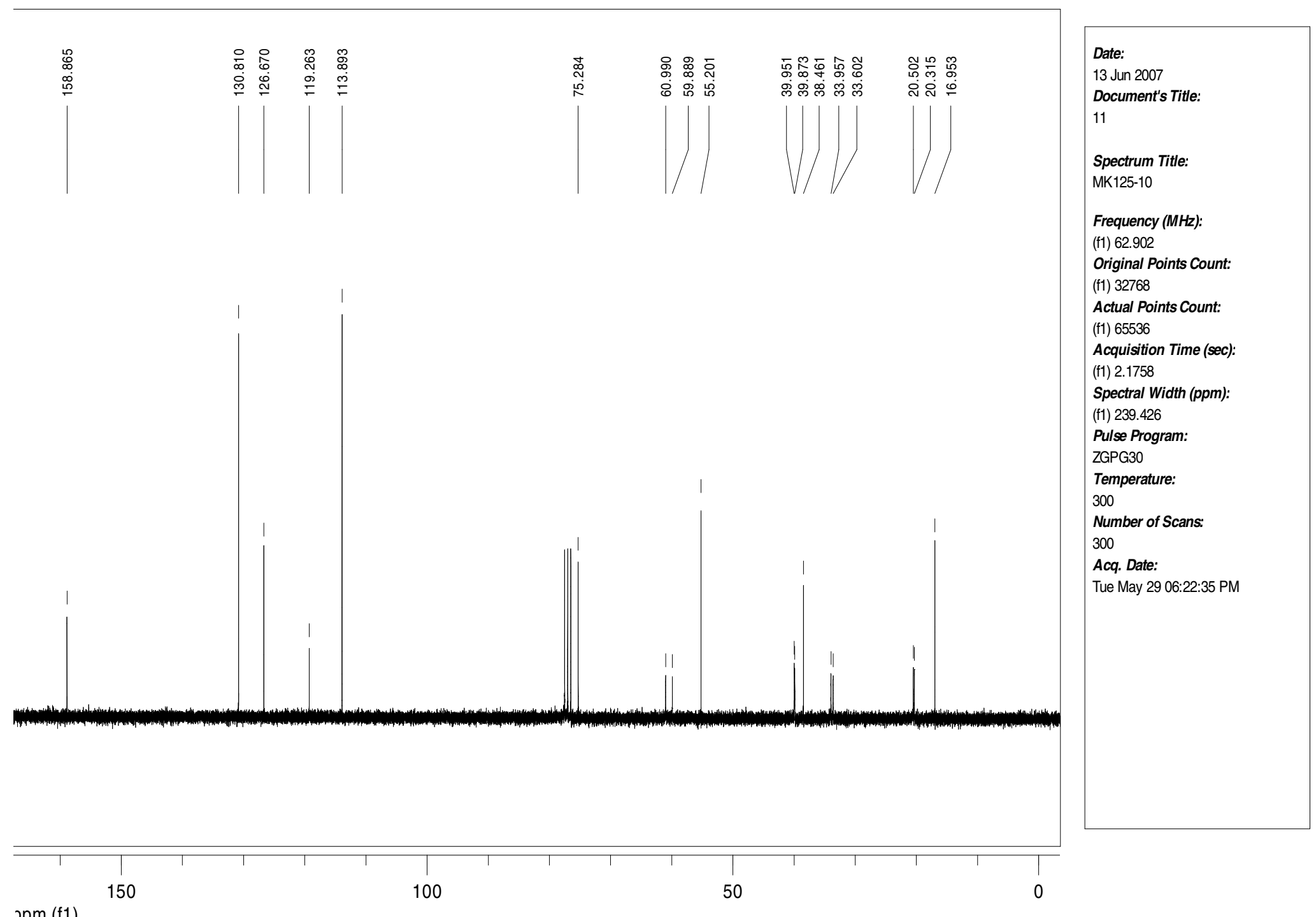


3-(4-Fluoro-phenyl)- $N, N$-dimethyl-2-(2,2,6,6-tetramethyl-piperidin-1-yloxy)-propionamide (4k)

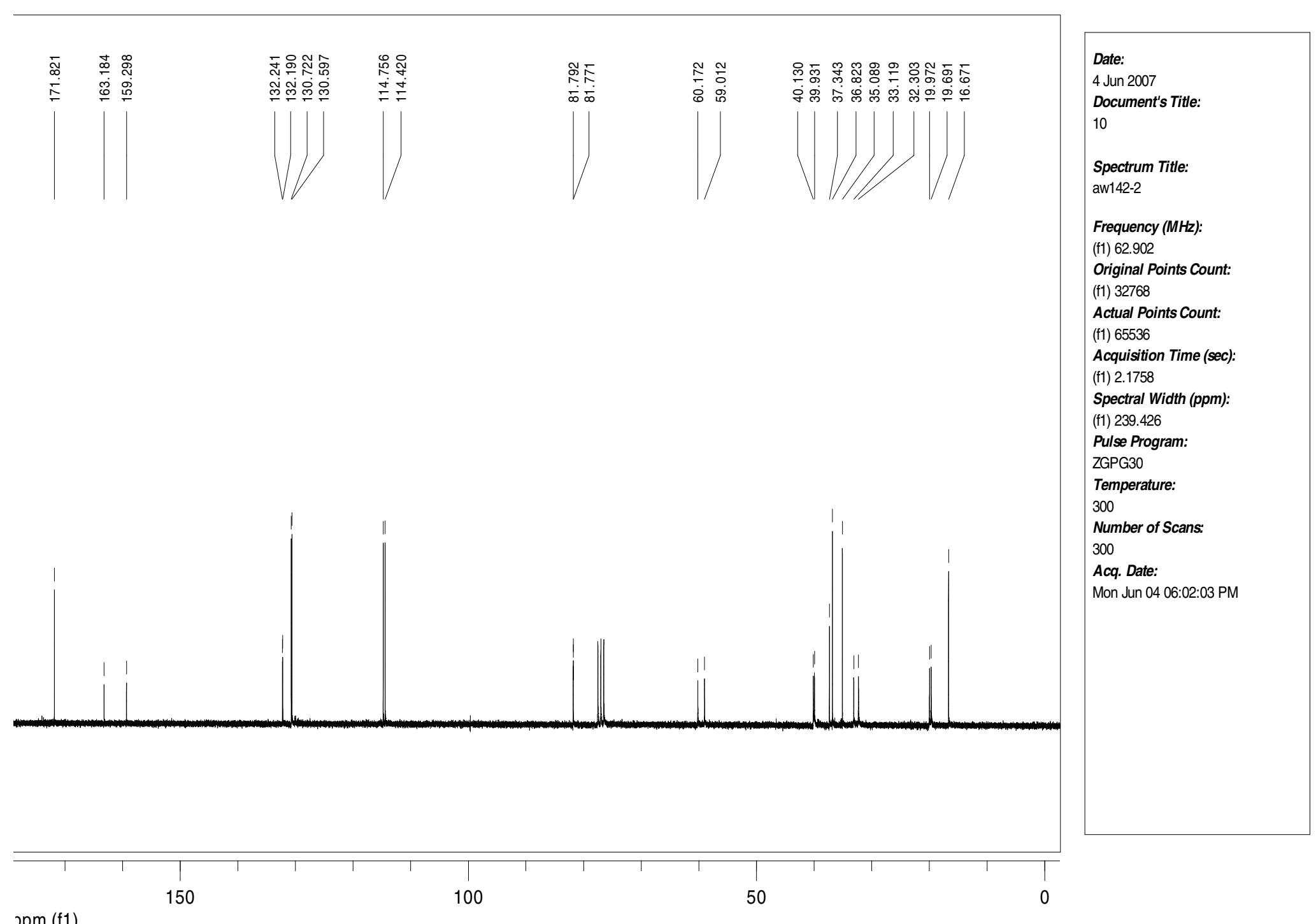


1-[2-(4-Methoxy-phenyl)-1-phenyl-ethoxy]-2,2,6,6-tetramethyl-piperidine (4I)

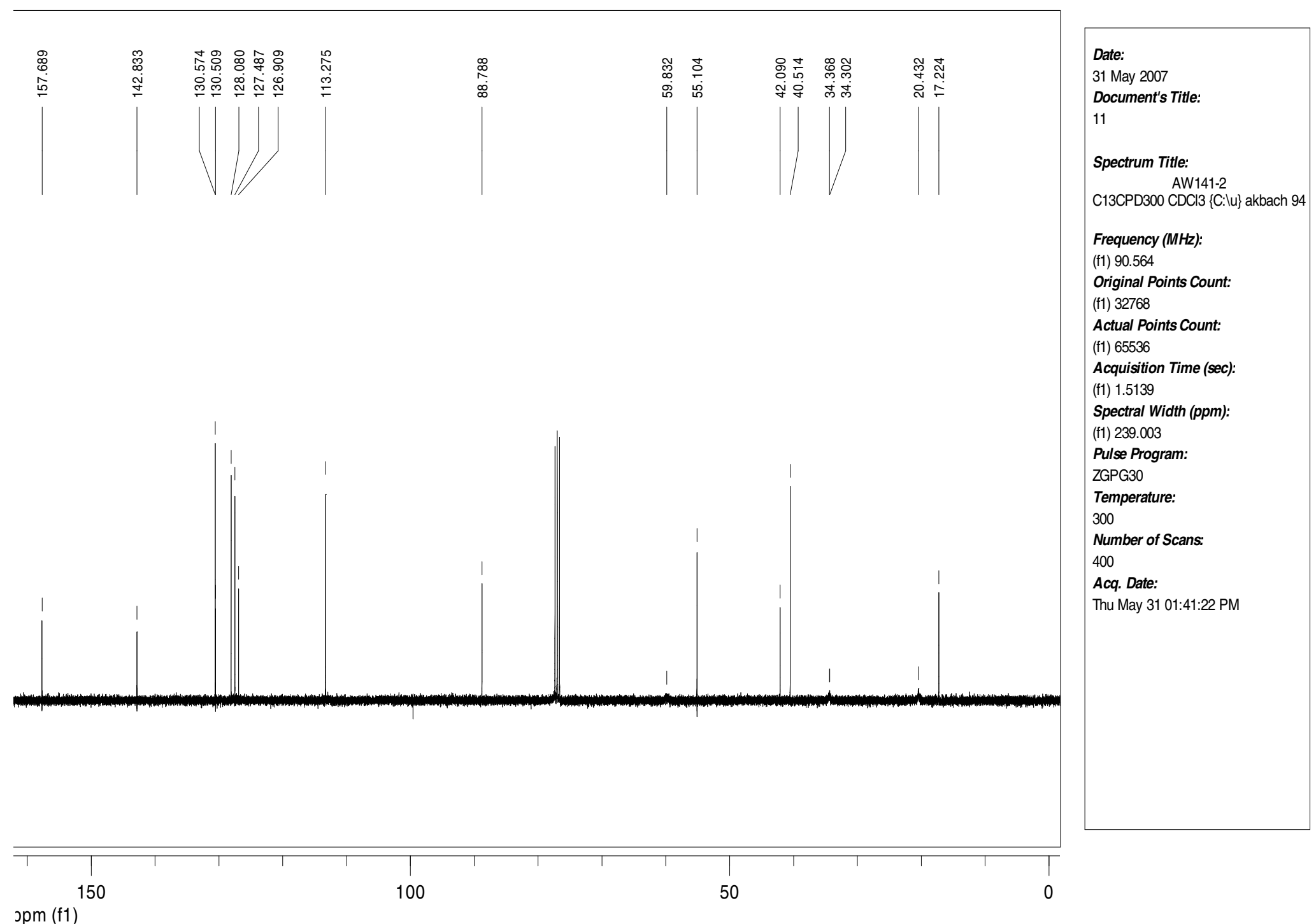


trans-4-(4-Methoxy-phenyl)-5-(2,2,6,6-tetramethyl-piperidin-1-yloxy)-[1,3]dioxolan-2-one (4m)

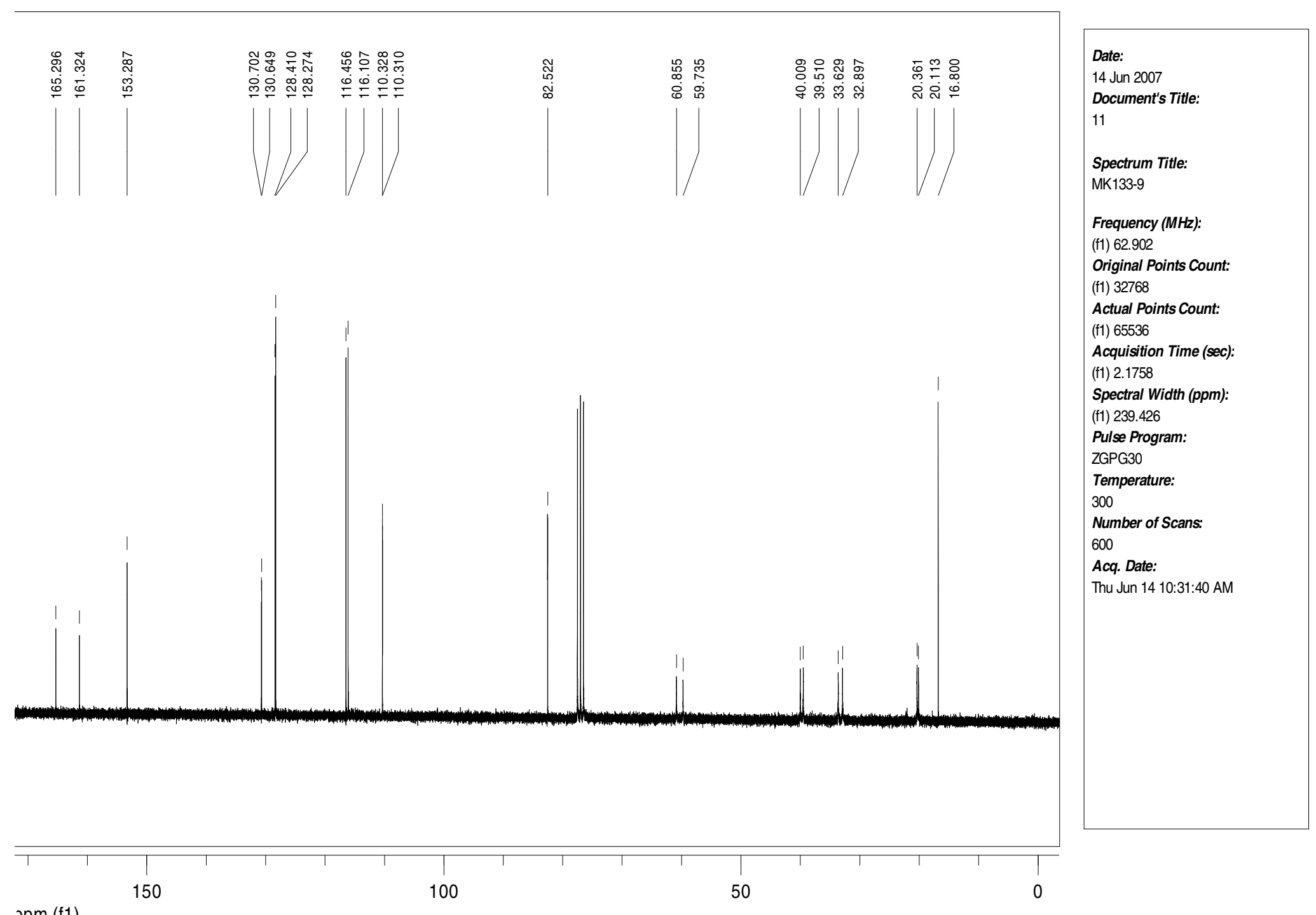


cis-4-(4-Methoxy-phenyl)-5-(2,2,6,6-tetramethyl-piperidin-1-yloxy)-[1,3]dioxolan-2-one (4m')

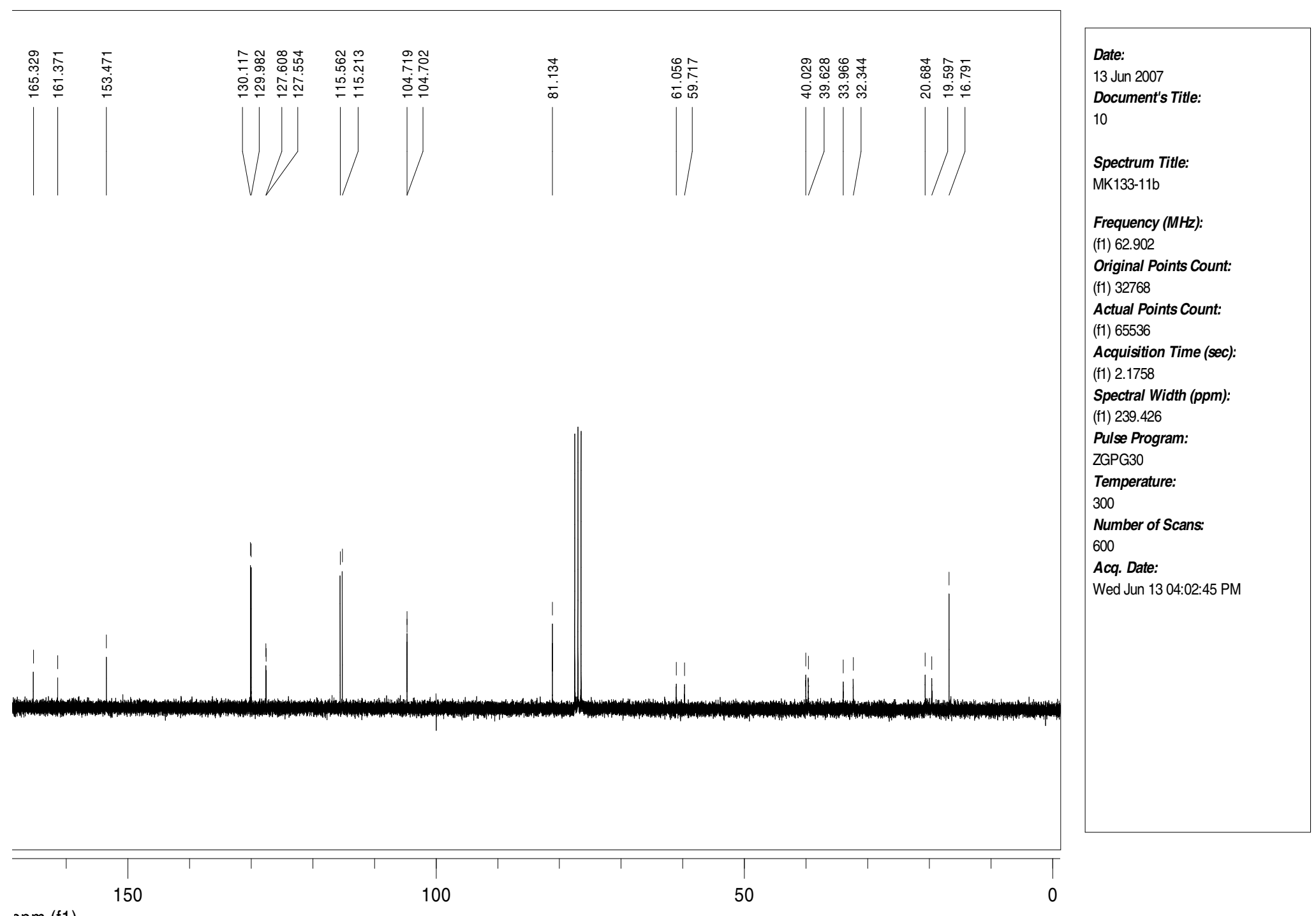


2-(4-Methoxy-phenyl)-1-(2,2,6,6-tetramethyl-piperidin-1-yloxy)-cyclohexanecarboxylic acid methyl ester (4n)

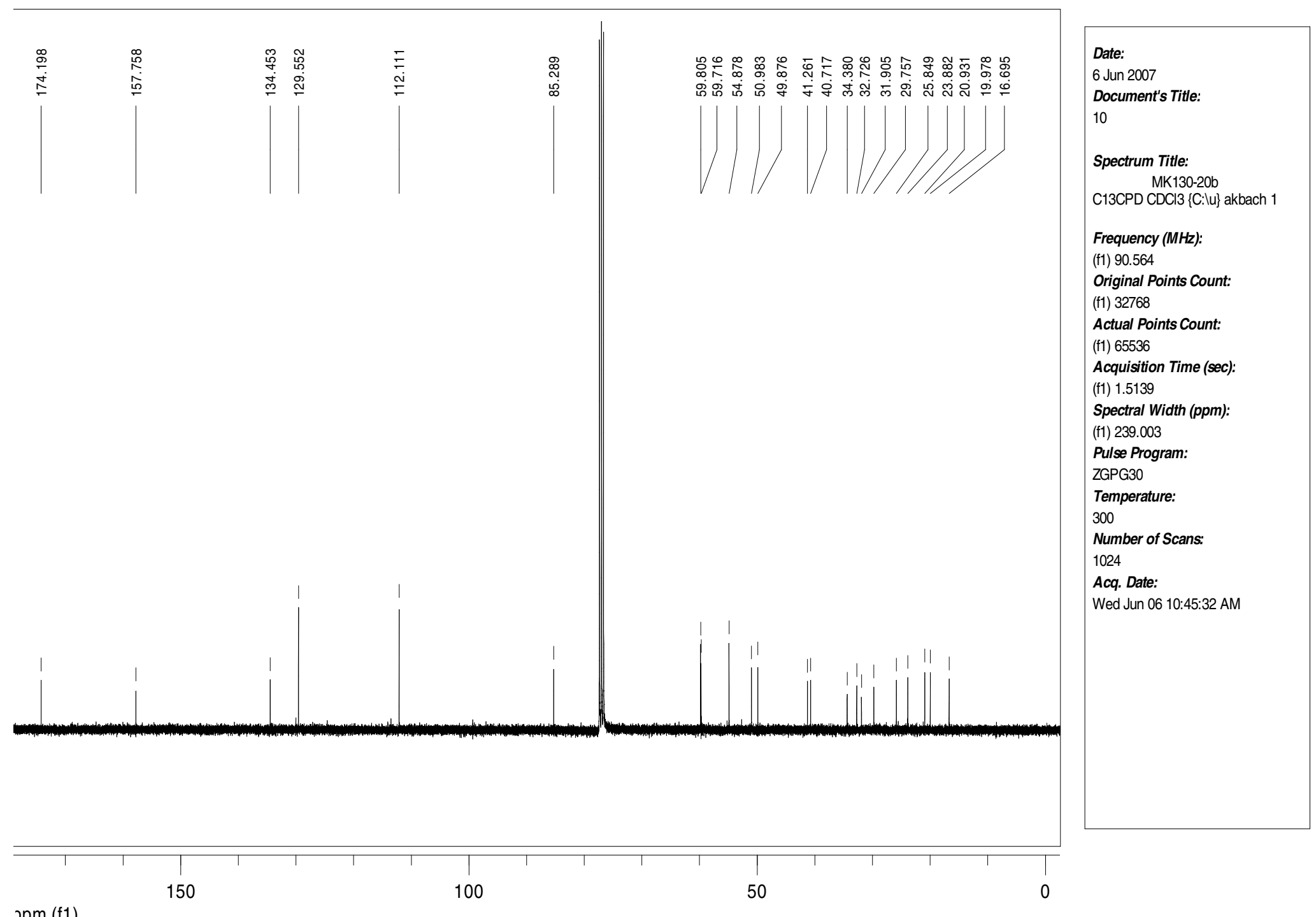


2-(4-Fluoro-phenyl)-1-phenyl-2-(2,2,6,6-tetramethyl-piperidin-1-yloxy)-ethanone (5)

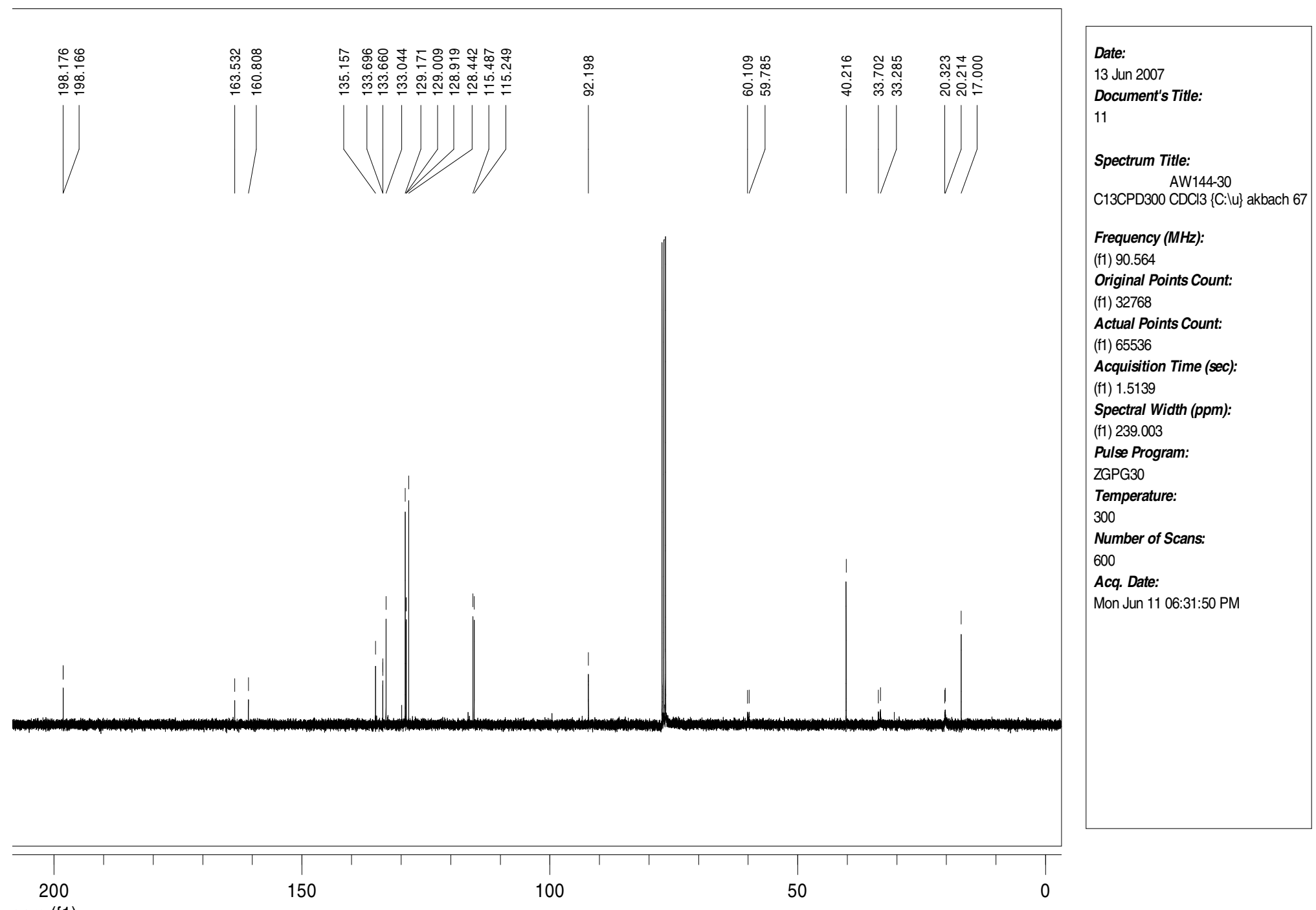


Acetic acid 1-acetoxymethyl-2-(4-methoxy-phenyl)-ethyl ester (6)

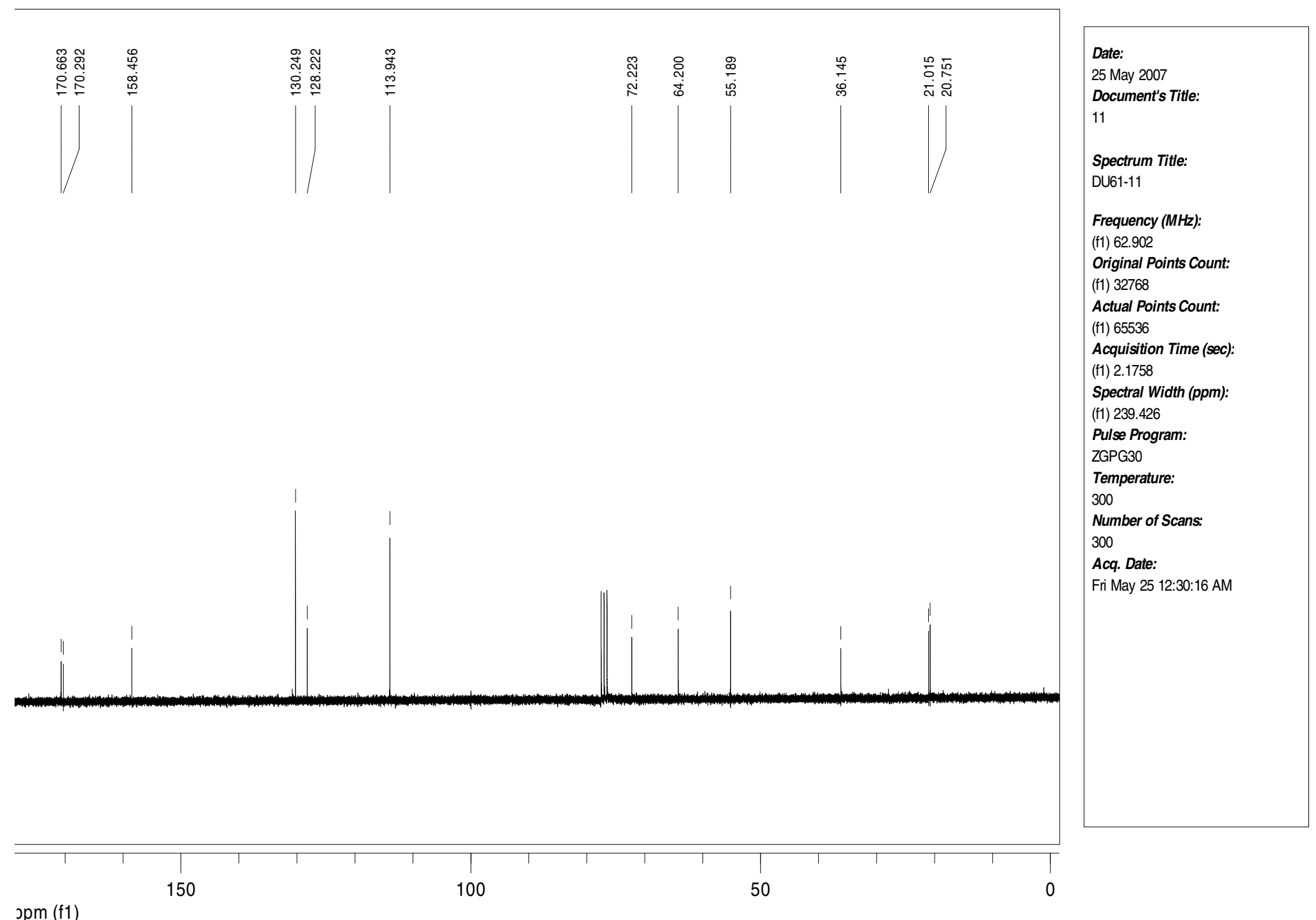




\section{1-Oxo-isochroman-3-carboxylic acid ethyl ester (7)}

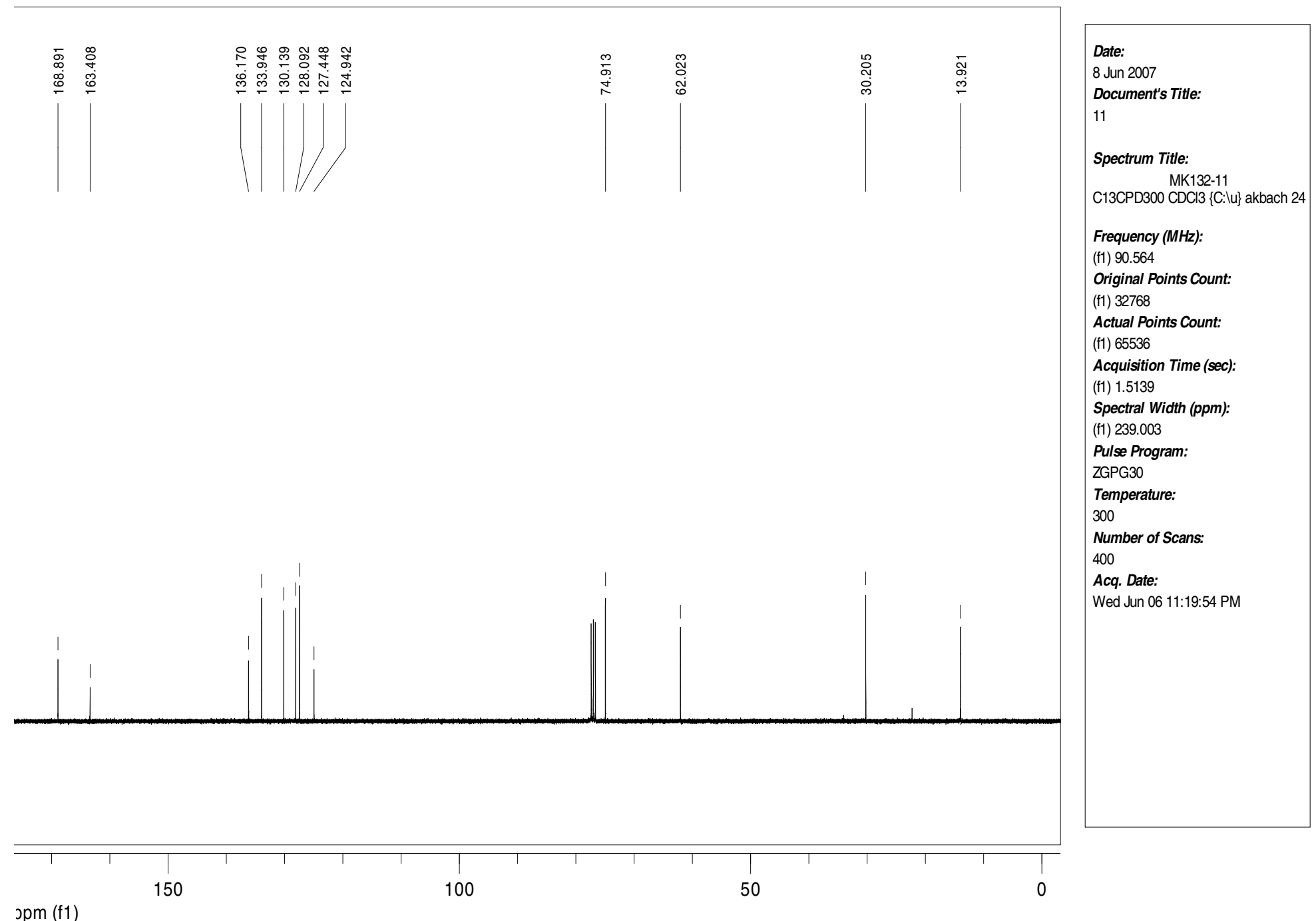

